\title{
Limnological characteristics of 56 lakes in the Central Canadian Arctic Treeline Region
}

\author{
Kathleen M. RÜHLAND*, John P. SMOL, Xiaowa WANG ${ }^{1)}$ and Derek C.G. MUIR ${ }^{1)}$ \\ Paleoecological Environmental Assessment and Research Laboratory (PEARL), Department of Biology, Queen's University, \\ Kingston, Ontario, Canada, K7L 3N6 \\ ${ }^{1)}$ National Water Research Institute, Canada Centre for Inland Waters, Burlington, Ontario, Canada, L7R 4A6 \\ *e-mail corresponding author: 3kmr5@biology.queensu.ca
}

\begin{abstract}
Measured environmental variables from 56 lakes across the Central Canadian Treeline Region exhibited clear limnological differences among subpolar ecozones, reflecting strong latitudinal changes in biome characteristics (e.g. vegetation, permafrost, climate). Principal Components Analysis (PCA) clearly separated forested sites from tundra sites based on distinct differences in limnological characteristics. Increases in major ions and related variables (e.g. dissolved inorganic carbon, DIC) were higher in boreal forest sites in comparison to arctic tundra sites. The higher values recorded in the boreal forest lakes may be indirectly related to differences in climatic factors in these zones, such as the degree of permafrost development, higher precipitation and runoff, duration of ice-cover on the lakes, and thicker and better soil development. Similar to trends observed in DIC, substantially higher values for dissolved organic carbon (DOC) were measured in boreal forest lakes than in arctic tundra lakes. This was likely due to higher amounts of catchment-derived DOC entering the lakes from coniferous leaf litter sources. Relative to arctic tundra lakes, boreal forest lakes had higher nutrient concentrations, particularly total nitrogen (TN), likely due to warmer conditions, a longer growing season, and higher precipitation, which would enhance nutrient cycling and primary productivity. Results suggest that modern aquatic environments at opposite sides of the central Canadian arctic treeline (i.e. boreal forest and arctic tundra) exhibit distinct differences in water chemistry and physical conditions. These limnological trends may provide important information on possible future changes with additional warming.
\end{abstract}

Key words: arctic treeline, climatic change, dissolved inorganic carbon, dissolved organic carbon, major ions, nutrients

\section{INTRODUCTION}

Circumpolar treeline regions are typically characterized by sharp changes in climate and vegetation. These steep gradients are often reflected by changes in the physical and chemical properties of aquatic systems as one crosses from forested to tundra environments (e.g. Pienitz et al. 1997a, b). Changes in climate are predicted to be most pronounced at these higher latitude regions, as suggested by General Circulation Models (GCMs) (Foley et al. 1994; Bonan et al. 1995) as well as by paleoecological data (Overpeck et al. 1997; Mann et al. 1999). These aquatic ecosystems will likely be affected both directly (e.g. water temperature increases, changes in precipitation/evaporation ratios) and indirectly (e.g. increased duration of ice-free season, change in the length and stability of lakewater stratification, permafrost thawing, and changes in the distribution of catchment vegetation) by climatic warming (Slaughter et al. 1995; Rouse et al. 1997; Schindler 1997; Serreze et al. 2000).

Limnological studies in the Central Canadian Arctic Treeline Region are still relatively rare (Healey \& Woodall 1973; Welch \& Legault 1986; Pienitz et al. 1997a; Rühland \& Smol 1998) and point to a need to extend these studies within this relatively unknown but important ecotonal region. Due to the vastness of the treeline region of Canada's Northwest and Nunavut Territories, it is not surprising that distinct differences in physical settings exist (e.g. local geology, hydrology, etc.). As a consequence, lakewater chemistry can be quite variable both within and among the different vegetational/climatic ecozones. These dissimilarities were highlighted in a previous study (Rühland \& Smol 1998) where the 70 study lakes spanned two distinct geological provinces (Bear and Slave provinces) resulting in differences in both chemistry and aquatic biota (diatoms). In this present study, our lakes represent a transect northeast from this previous study, and as a result is entirely contained within the Slave Geological Province, thereby minimizing the influence of geology, and maximizing any climatic changes along the vegetational gradient.

Comparisons of limnological studies carried out across the circumpolar treeline region has highlighted many similarities as well as differences in the trends and relationships in lakewater chemistry from region to region. For example, major cations (e.g. calcium, sodium, magnesium, potassium) and related variables (alkalinity, DIC, $\mathrm{pH}$ ) were found to increase along latitudinal transects from arctic tundra to boreal forest catchments in most regions with the exception of Fennoscandia (Weckström et al. 1997a, b; Blom et al. 1999) and the 


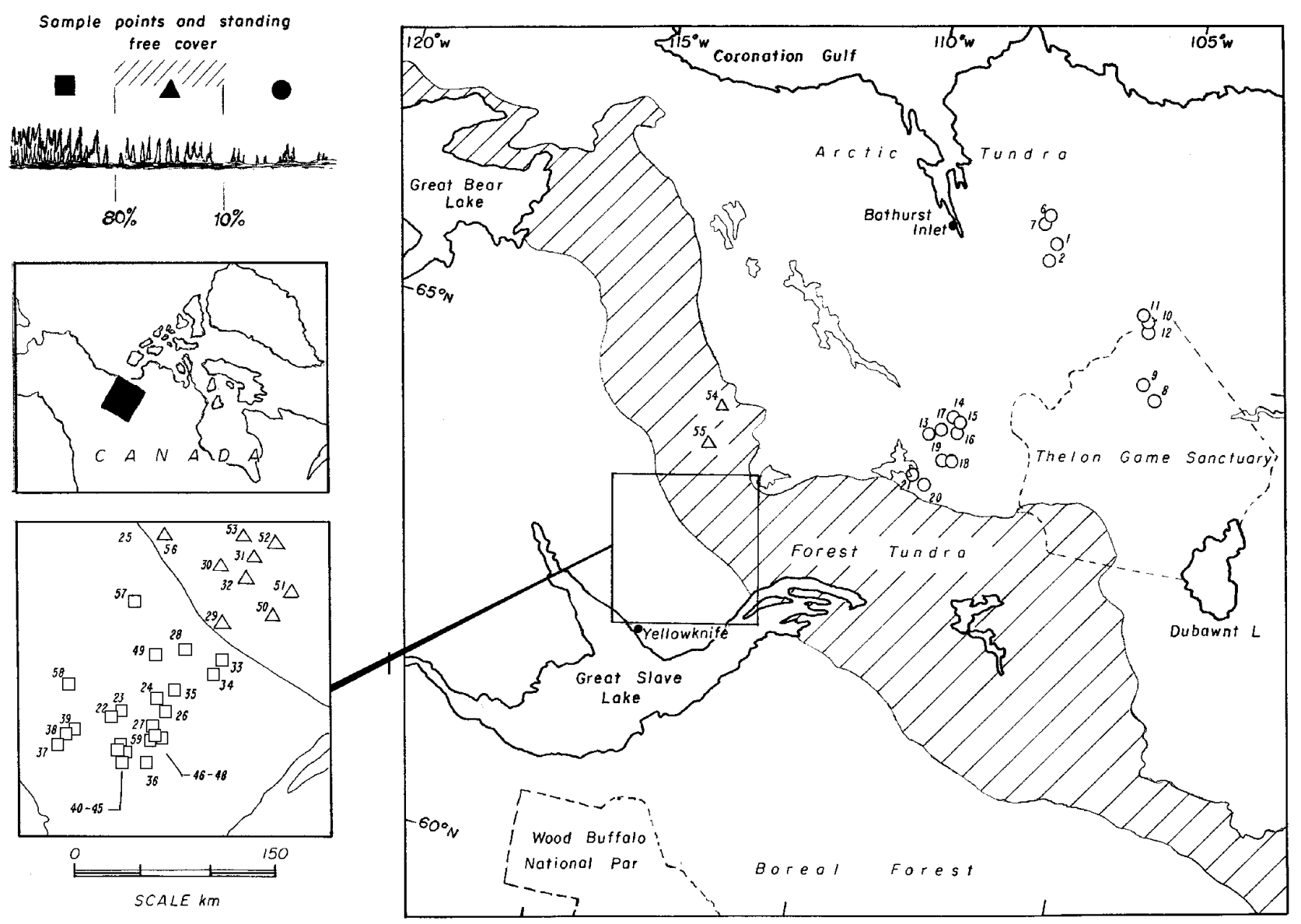

Fig. 1. Location of the 56 study lakes showing their distribution across the three vegetational zones (boreal forest, forest-tundra, and arctic tundra).

Lena and Pechora regions of Siberia (Duff et al. 1998). These inconsistencies were likely related to differences in geology, hydrology, human impact, physiographic relief, proximity to the ocean, vegetation, and climate. Similarly, changes in DOC and in nutrient relationships have also been reported (where measured) to be closely related to catchment vegetation (Pienitz 1993) and availability of phosphorus from bedrock materials (Downing \& McCauley 1992; Hamilton et al. 2001). However, given the vastness of subarctic and arctic regions (approximately six million $\mathrm{km}^{2}$ ) in North America (Rouse et al. 1997), coupled with the paucity of limnological studies, it is important that research continues in these remote regions to contribute to our understanding of the natural variability of these sensitive freshwater ecosystems and their responses to environmental changes.

This paper explores the relationships between chemical and physical properties of 56 lakes and 27 environmental variables within the central treeline region in Canada's Nunavut and Northwest Territories. Unlike our previous paper (Rühland \& Smol 1998), this study includes important additional limnological measurements including nutrients (e.g. TN, total phosphorus
(TP), DIC, and DOC). As the results reported here are part of a more extensive paleoecological study that examines the relationship between diatom distributions and these environmental gradients (Rühland \& Smol 2002; Rühland et al. 2003), these data are essential for a better understanding of some of the fundamental differences among these arctic lakes, especially as these variables are of great importance to aquatic biota. Our results show that spatial gradients (e.g. temperature, precipitation, vegetation, permafrost) across the central Canadian treeline were often closely reflected in the lakewater chemistry and that distinct limnological differences exist among boreal ecozones. The clear relationships observed among ecozone characteristics and limnological variability along the climatic/vegetational gradient reported here will be compared to other limnological studies of northern treeline regions.

\section{STUDY AREA}

The 56 study lakes were sampled along a southwest to northeast transect from the Yellowknife area $\left(62^{\circ} 32.72^{\prime} \mathrm{N}, 114^{\circ} 07.13^{\prime} \mathrm{W}\right)$ towards Bathurst Inlet $\left(66^{\circ} 41.3^{\prime} \mathrm{N}, 104^{\circ} 55.9^{\prime} \mathrm{W}\right)$ in the central Canadian Subarctic (Fig. 1, Tab. 1). This lakeset crosses strong 
Tab. 1. Geographical co-ordinates and vegetational zonations of the 56 study sites. - = Arctic Tundra, $\Delta=$ Forest-Tundra, $\bullet=$ Boreal Forest.

\begin{tabular}{|c|c|c|c|c|c|c|c|}
\hline & SITE & $\mathrm{LAT}(\mathrm{N})$ & LONG(W) & & SITE & LAT(N) & $\mathrm{LONG}(\mathrm{W})$ \\
\hline & TK-1 & $66^{\circ} 28.58^{\prime}$ & $104^{\circ} 49.52^{\prime}$ & $\Delta$ & TK-32 & $63^{\circ} 29.05^{\prime}$ & $112^{\circ} 12.63^{\prime}$ \\
\hline & TK-2 & $66^{\circ} 20.90^{\prime}$ & $104^{\circ} 56.45^{\prime}$ & - & TK-33 & $63^{\circ} 12.97^{\prime}$ & $112^{\circ} 36.05^{\prime}$ \\
\hline & TK-6 & $66^{\circ} 41.31^{\prime}$ & $104^{\circ} 55.86^{\prime}$ & - & TK-34 & $63^{\circ} 07.64^{\prime}$ & $112^{\circ} 47.67^{\prime}$ \\
\hline & TK-7 & $66^{\circ} 41.23^{\prime}$ & $104^{\circ} 55.89^{\prime}$ & - & TK-35 & $63^{\circ} 02.72^{\prime}$ & $113^{\circ} 06.46^{\prime}$ \\
\hline & TK-8 & $64^{\circ} 41.28^{\prime}$ & $102^{\circ} 26.42^{\prime}$ & - & TK-36 & $62^{\circ} 45.75^{\prime}$ & $113^{\circ} 28.63^{\prime}$ \\
\hline & TK-9 & $64^{\circ} 53.37^{\prime}$ & $102^{\circ} 45.07^{\prime}$ & - & TK-37 & $62^{\circ} 32.72^{\prime}$ & $114^{\circ} 07.13^{\prime}$ \\
\hline & TK-10 & $65^{\circ} 29.42^{\prime}$ & $103^{\circ} 22.01^{\prime}$ & - & TK-38 & $62^{\circ} 33.59^{\prime}$ & $114^{\circ} 01.34^{\prime}$ \\
\hline & TK-11 & $65^{\circ} 33.62^{\prime}$ & $103^{\circ} 23.25^{\prime}$ & - & TK-39 & $62^{\circ} 32.89^{\prime}$ & $113^{\circ} 57.13^{\prime}$ \\
\hline & TK-12 & $65^{\circ} 22.50^{\prime}$ & $103^{\circ} 33.45^{\prime}$ & - & TK-40 & $62^{\circ} 32.87^{\prime}$ & $113^{\circ} 56.09^{\prime}$ \\
\hline & TK-13 & $64^{\circ} 36.15^{\prime}$ & $107^{\circ} 26.23^{\prime}$ & - & TK-41 & $62^{\circ} 34.09^{\prime}$ & $114^{\circ} 01.74^{\prime}$ \\
\hline & TK-14 & $64^{\circ} 35.90^{\prime}$ & $107^{\circ} 05.93^{\prime}$ & - & TK-42 & $62^{\circ} 32.75^{\prime}$ & $113^{\circ} 53.40^{\prime}$ \\
\hline & TK-15 & $64^{\circ} 35.74^{\prime}$ & $107^{\circ} 05.49^{\prime}$ & - & TK-43 & $62^{\circ} 31.49^{\prime}$ & $113^{\circ} 50.20^{\prime}$ \\
\hline & TK-16 & $64^{\circ} 35.60^{\prime}$ & $107^{\circ} 05.30^{\prime}$ & - & TK-44 & $62^{\circ} 31.49^{\prime}$ & $113^{\circ} 50.20^{\prime}$ \\
\hline & TK-17 & $64^{\circ} 35.69^{\prime}$ & $107^{\circ} 05.01^{\prime}$ & - & TK-45 & $62^{\circ} 31.01^{\prime}$ & $113^{\circ} 46.76^{\prime}$ \\
\hline & TK-18 & $64^{\circ} 09.39^{\prime}$ & $107^{\circ} 49.48^{\prime}$ & - & TK-46 & $62^{\circ} 30.44^{\prime}$ & $113^{\circ} 39.91^{\prime}$ \\
\hline & TK-19 & $64^{\circ} 09.39^{\prime}$ & $107^{\circ} 49.48^{\prime}$ & - & TK-47 & $62^{\circ} 40.69^{\prime}$ & $113^{\circ} 51.03^{\prime}$ \\
\hline & TK-20 & $64^{\circ} 09.00^{\prime}$ & $107^{\circ} 49.00^{\prime}$ & - & TK-48 & $62^{\circ} 48.27^{\prime}$ & $113^{\circ} 26.12^{\prime}$ \\
\hline & TK-21 & $64^{\circ} 09.00^{\prime}$ & $107^{\circ} 49.00^{\prime}$ & - & TK-49 & $63^{\circ} 10.99^{\prime}$ & $112^{\circ} 22.83^{\prime}$ \\
\hline & TK-22 & $62^{\circ} 42.03^{\prime}$ & $113^{\circ} 59.19^{\prime}$ & $\Delta$ & TK-50 & $63^{\circ} 18.48^{\prime}$ & $111^{\circ} 58.27^{\prime}$ \\
\hline & TK-23 & $62^{\circ} 43.73^{\prime}$ & $113^{\circ} 49.53^{\prime}$ & $\Delta$ & TK-51 & $63^{\circ} 32.91^{\prime}$ & $111^{\circ} 42.34^{\prime}$ \\
\hline & TK-24 & $62^{\circ} 54.04^{\prime}$ & $113^{\circ} 13.15^{\prime}$ & $\Delta$ & TK-52 & $63^{\circ} 35.61^{\prime}$ & $112^{\circ} 25.12^{\prime}$ \\
\hline & TK-25 & $63^{\circ} 04.81^{\prime}$ & $113^{\circ} 02.88^{\prime}$ & $\Delta$ & TK-53 & $63^{\circ} 42.54^{\prime}$ & $112^{\circ} 34.40^{\prime}$ \\
\hline & TK-26 & $62^{\circ} 54.14^{\prime}$ & $113^{\circ} 23.22^{\prime}$ & $\Delta$ & TK-54 & $64^{\circ} 30.68^{\prime}$ & $112^{\circ} 41.47^{\prime}$ \\
\hline & TK-27 & $62^{\circ} 49.23^{\prime}$ & $113^{\circ} 33.75^{\prime}$ & $\Delta$ & TK-55 & $64^{\circ} 06.01^{\prime}$ & $113^{\circ} 01.54^{\prime}$ \\
\hline - & TK-28 & $63^{\circ} 15.31^{\prime}$ & $113^{\circ} 00.22^{\prime}$ & $\Delta$ & TK-56 & $63^{\circ} 41.42^{\prime}$ & $113^{\circ} 26.68^{\prime}$ \\
\hline$\Delta$ & TK-29 & $63^{\circ} 25.25^{\prime}$ & $112^{\circ} 39.81^{\prime}$ & $\Delta$ & TK-57 & $63^{\circ} 20.21^{\prime}$ & $113^{\circ} 42.30^{\prime}$ \\
\hline$\Delta$ & TK-30 & $63^{\circ} 33.70^{\prime}$ & $112^{\circ} 21.21^{\prime}$ & - & TK-58 & $62^{\circ} 53.37^{\prime}$ & $114^{\circ} 04.24^{\prime}$ \\
\hline$\Delta$ & TK-31 & $63^{\circ} 37.03^{\prime}$ & $112^{\circ} 17.77^{\prime}$ & - & TK-59 & $62^{\circ} 30.19^{\prime}$ & $113^{\circ} 38.20^{\prime}$ \\
\hline
\end{tabular}

vegetational and climatic boundaries from the boreal forest ecozone in the southwest to the arctic tundra ecozone in the northeast (Fig. 1). The region is situated on Canadian Shield bedrock consisting of gneiss, migmatite, granitoid intrusions, and metamorphosed volcanic and sedimentary rocks (Padgham \& Fyson 1992). The entire study region is underlain by permafrost and ranges from discontinuous permafrost in the northern boreal forest to continuous permafrost in the arctic tundra zone and thus the boundary approximately follows the position of treeline. The soils are poorly developed as a consequence of the Pleistocene glaciation and from permafrost underlying the region today (Clayton et al. 1977).

Temperature and precipitation change sharply from the southern to the northern reaches of the study area, with cooler and drier conditions towards Coronation Gulf (mean January night-time temperatures of -37.5 ${ }^{\circ} \mathrm{C}$, mean July day-time temperatures of $12.5^{\circ} \mathrm{C}$, and annual precipitation of $200 \mathrm{~mm}$ ) and warmer, moister conditions towards Yellowknife (mean January nighttime temperatures of $-30{ }^{\circ} \mathrm{C}$, mean July day-time temperatures of $22.5^{\circ} \mathrm{C}$, and annual precipitation of 267 mm) (Environment Canada 1990; Canada 1991). Lakes are frozen for most of the year with the average openwater period ranging from 90 days at the northernmost locale to 120 days for sites near Yellowknife (Canada 1991).
Three main vegetation zones were recognized, following the vegetational distributions presented by Timoney et al. (1993) and Ritchie (1993). These ecozones were delineated according to "percentage treecover" derived from field observations, topographic maps, aerial photographs, and maps derived from satellite imagery (Canada 1996). The forest-tundra transition zone (High Subarctic) lies between $10 \%$ and $80 \%$ standing tree cover and is characterized by Picea mariana (black spruce), P. glauca (white spruce), and Larix laricuna (larch) (Sirois 1992). The boreal forest (Low Subarctic) lies south of $80 \%$ standing tree cover and is characterized by closed-crown, conifer-dominated forest. Picea mariana, P. glauca, Abies balsamea (balsam fir), and Pinus banksiana (jackpine) are the most common conifers (Ritchie 1993). The arctic tundra (Low Arctic) lies north of $10 \%$ standing tree cover and consists predominantly of dwarf shrub vegetation including Betula glandulosa (resin birch), Salix (willow) species, and species of heath shrubs (e.g. Arctostaphylos, Ledum, and Vaccinium) (Ritchie 1993).

\section{MATERIALS AND METHODS}

\subsection{Sample Collection and Environmental Data}

Lakes were selected within each ecozone with the criteria that the lakes chosen were approximately similar in size and morphometry so that trends observed in en- 
vironmental variables along the latitudinal transect would likely reflect changes in ecozone characteristics. Samples were collected between 7 August and $19 \mathrm{Au}-$ gust 1996 for TK-1 to TK-36, and between 14 August and 23 August 1998 for TK-37 to TK-59. All lakes are unnamed and so are referred to numerically. The final lakeset consisted of 18 arctic tundra sites, 11 forest-tundra sites and 27 boreal forest sites for a total of 56 lakes (Tab. 1, Fig. 1). As many of these lakes were located in remote areas, the sites were sampled only once and therefore represent a "snapshot" of limnological conditions measured at approximately the same time of the year in two different years.

Limnological measurements were taken from the deepest part of the lake (usually at the lake centre) from an inflatable boat or off the pontoons of a helicopter. Water depth was measured using a weighted graduated rope. Where possible, temperature and conductivity measurements were taken at the surface and at the bottom of the lake using a Yellow Springs Instrument (YSI model 33) salinity, conductivity, temperature meter. Multiple readings for water temperature were taken using hand-held thermometers. Water transparency was determined using a $22 \mathrm{~cm}$ diameter Secchi disk. At each lake, several $\mathrm{pH}$ measurements were taken using handheld meters (Hanna) that were frequently calibrated to standard buffers. Due to equipment problems, $\mathrm{pH}$ for several lakes were measured shortly after sampling at DIAND laboratories in Yellowknife (TK-47 to TK-59). Lake surface area (AREA) was determined from a series of topographic maps $(1: 50,000)$ that were scanned and converted into digital format using the computer program Jandel Sigmascan (v. 3.0).

Water chemistry samples were collected at approximately $30 \mathrm{~cm}$ beneath the water surface and were treated in the field according to procedures outlined in the Environment Canada (1994a, b) Analytical Methods Manual. Water samples for major ions and trace metals were collected in $125 \mathrm{ml}$ acid pre-cleaned Nalgene bottles. Nutrient analyses (total nitrogen and total phosphorus) was filtered through Sartorius ${ }^{\circledR}$ (cellulose acetate) filters (pore size $=0.45 \mu \mathrm{m}$ ) and stored in $100 \mathrm{ml}$ glass bottles. For particulate organic carbon (POC) and particulate organic nitrogen (PON), 100 to $400 \mathrm{ml}$ of water was filtered through Whatman ${ }^{\circledR}$ pre-ignited glass microfibre filters (pore size $=0.45 \mu \mathrm{m}$ ). For chlorophyll- $a$ (corrected and uncorrected for phaeophytin), a measured volume of water (200 to $500 \mathrm{ml}$ ) was filtered through Whatman ${ }^{\circledR}$ glass microfibre filters (pore size $=$ $0.45 \mu \mathrm{m})$. The filters for POC, PON and chlorophyll- $a$ were folded in half, placed inside plastic Petri dishes, and then wrapped in aluminum foil. The filters and water samples were packed inside storage coolers, and then transported to the National Water Research Institute (Burlington, Ontario) for analyses.

In addition to the environmental measurements mentioned above, the closest distance to $80 \%$ standing treecover (DIST) was measured for each site. Distance from treeline was measured from the northern limit of the boreal forest zone. The forest-tundra transitional zone (High Subarctic) in this study region occurs between $10 \%$ and $80 \%$ standing treecover, the arctic tundra zone (Low Arctic) lies north of $10 \%$ standing treecover, and the boreal forest zone (Low Subarctic) lies south of $80 \%$ standing treecover.

For each lake, 40 chemical and 6 physical/geographical variables were measured. These variables consisted of total phosphorus filtered (TPF), total phosphorus unfiltered (TPUF), total nitrogen (TN), soluble reactive phosphatephosphorus (SRP), total Kjeldahl nitrogen (TKN), ammonia $\left(\mathrm{NH}_{3}\right)$, nitrite $\left(\mathrm{NO}_{2}\right)$, nitrite-nitrate $\left(\mathrm{NO}_{2}-\mathrm{NO}_{3}\right)$, dissolved organic carbon (DOC), dissolved inorganic carbon (DIC), $\mathrm{pH}(\mathrm{pH})$, conductivity (COND), particulate organic carbon (POC), particulate organic nitrogen $(\mathrm{PON})$, chlorophyll- $a$ corrected (CHLA-corr), chlorophyll- $a$ uncorrected (CHLA), dissolved silica $\left(\mathrm{SiO}_{2}\right)$, calcium $(\mathrm{Ca})$, sodium $(\mathrm{Na})$, magnesium $(\mathrm{Mg})$, potassium $(\mathrm{K})$, chloride $(\mathrm{Cl})$, sulphate $\left(\mathrm{SO}_{4}\right)$, lithium $(\mathrm{Li})$, barium $(\mathrm{Ba})$, strontium $(\mathrm{Sr})$, silver $(\mathrm{Ag})$, aluminum $(\mathrm{Al})$, beryllium $(\mathrm{Be})$, cadmium $(\mathrm{Cd})$, cobalt $(\mathrm{Co})$, chromium $(\mathrm{Cr})$, copper $(\mathrm{Cu})$, iron $(\mathrm{Fe})$, manganese $(\mathrm{Mn})$, molybdenum $(\mathrm{Mo})$, nickel $(\mathrm{Ni})$, lead $(\mathrm{Pb})$, vanadium $(\mathrm{V})$, and zinc $(\mathrm{Zn})$. Physical variables measured on site consisted of maximum lake depth (DEPTH), secchi disk depth (SECCHI), and surface water temperature (TEMP). Geographical variables included distance from treeline (DIST), latitude (LAT), and lake surface area (AREA).

\subsection{Data Screening and Analysis}

A total of 19 of the original 46 measured variables had numerous sites (19 to 56 sites) with values below the level of detection (BDL). These variables included $\mathrm{Ag}, \mathrm{Be}, \mathrm{Cd}, \mathrm{Co}, \mathrm{Cr}, \mathrm{Cu}, \mathrm{Mo}, \mathrm{Ni}, \mathrm{Pb}, \mathrm{V}, \mathrm{Zn}, \mathrm{NH}_{3}, \mathrm{NO}_{2}-$ $\mathrm{NO}_{3}, \mathrm{NO}_{2}, \mathrm{CHLA}$-corr, and SRP. POC and PON also fall under this category as 23 of the lakes did not have this information available. Secchi disk depth was also eliminated from the dataset as numerous shallow lakes were clear to the bottom and therefore did not contribute analytically meaningful measurements (see Cumming et al. 1995). Three variables $(\mathrm{Na}, \mathrm{Mn}$, and $\mathrm{Li}$ ) were run passively in the statistical analyses as they had fewer sites with values BDL (14-16 sites BDL). Total phosphorus filtered (TPF) displayed highly skewed distributions that could not be corrected through data transformations. However, as this was a potentially important variable, it was retained in the dataset and run passively in the statistical analyses. For all variables, sites that recorded several values below the detection limits were replaced with the measurement of detection for that particular variable (DIC $\left[0.1 \mathrm{mg} \mathrm{l}^{-1}\right]$ one site; Fe $[1.0 \mu \mathrm{g}$ $\left.\mathrm{l}^{-1}\right]$ one site; $\mathrm{K}\left[0.2 \mathrm{mg} \mathrm{l}^{-1}\right]$ two sites). For sites that had missing values (e.g. CHLA for boreal forest sites TK-39 and TK-48), the mean CHLA value for that ecozone was used to replace the missing measurements. The fi- 
nal environmental dataset consisted of 27 variables, 18 active chemical variables (TPUF, TN, TKN, CHLA, $\mathrm{SiO}_{2}, \mathrm{SO}_{4}, \mathrm{DIC}, \mathrm{DOC}, \mathrm{COND}, \mathrm{pH}, \mathrm{Ca}, \mathrm{Mg}, \mathrm{K}, \mathrm{Cl}, \mathrm{Al}$, $\mathrm{Ba}, \mathrm{Fe}$, and $\mathrm{Sr}$, 5 physical/geographical variables (DIST, LAT, AREA, DEPTH, and TEMP), and 4 passive variables (TPF, $\mathrm{Na}, \mathrm{Mn}$, and $\mathrm{Li}$ ).

The 27 environmental variables were tested for skewness and log transformed (with the exception of $\mathrm{pH}$ and TEMP) using the computer program CALIBRATE (Juggins \& ter Braak 1993). A Pearson correlation matrix was used to identify the strengths of relationships between these transformed environmental variables. Groups of environmental variables that were significantly correlated $(0.01 \geq p \leq 0.05)$ were identified through Bonferroni-adjusted probabilities.

Principal components analysis (PCA), a multivariate ordination technique, was used to interpret the major patterns of variation in the environmental data by examining the strengths of each variable in explaining the principal directions of variation for the 56 sites. Data were centred and standardized to mean zero and unit variance so that all environmental variables were comparable. PCA was carried out using the computer program CANOCO version 4.0 (ter Braak \& S Smilauer1998). The aim of this ordination analysis was to determine the interrelationships among the chemical and physical variables across the vegetational gradient. Therefore, the geographical variables (LAT and DIST) were treated as passive variables.

\section{RESULTS AND DISCUSSION}

Similar to other treeline limnological studies (e.g. Pienitz et al. 1997a, b; Weckström et al. 1997a, b; Rühland \& Smol 1998; Duff et al. 1998; Fallu \& Pienitz 1999; Gregory-Eaves et al. 2000; Fallu et al. 2002), our measured environmental variables followed terrestrial changes across the vegetational gradient. In general, values of major ions, nutrients, DIC, DOC, TEMP, $\mathrm{COND}, \mathrm{Ba}, \mathrm{Li}$, and $\mathrm{Sr}$ were highest for lakes in forested catchments and decreased with increasing latitude (Figs 2,3 , and 4). The majority of the measured variables were significantly different $(\mathrm{p}<0.05)$ between ecozones, in particular between the boreal forest and the arctic tundra zones (Tab. 2). The correlation matrix of the 27 environmental variables revealed high correlations among many of the measured variables (Tab. 3). As expected, the two geographic variables (DIST and LAT) were highly negatively correlated to most of the major and minor ions, nutrients, temperature, conductivity, DIC, and DOC. In contrast, $\mathrm{Si}, \mathrm{Fe}$, and $\mathrm{Al}$ were not significantly correlated to any of the other measured variables. Lake depth (negative correlation) and chlorophyll- $a$ (positive correlation) showed significant correlations only to nitrogen (TKN and TN). Although DOC and DIC were not significantly correlated to each other, they were correlated to many of the same variables (TEMP, COND, TN, K, Na, Ca, Mg, Sr, TKN, and Ba).
Unlike DOC, DIC was significantly and positively correlated to $\mathrm{pH}, \mathrm{TPF}, \mathrm{Li}$, and $\mathrm{Cl}$.

Tab. 2. Comparison of significant differences (two-tailed t-test results) of measured variables across the three vegetational zones [Arctic Tundra (AT), Forest-Tundra (FT), and Boreal Forest (BF)]. * - indicates significantly different $(\mathrm{p} \leq 0.05)$. Blank cells indicate there was no significant difference between ecozones. $\mathrm{TKN}=$ Total Kjeldahl Nitrogen. $\mathrm{TN}=$ Total Nitrogen unfiltered (particulate organic nitrogen $(\mathrm{PON})+\mathrm{TKN}+$ nitrite-nitrate $\left(\mathrm{NO}_{3} / \mathrm{NO}_{2}\right)$. TP-UF $=$ Total Phoshorus unfiltered.

\begin{tabular}{|c|c|c|c|}
\hline Variable & AT $v s$ FT & FT vs BF & $\mathrm{AT} v s \mathrm{BF}$ \\
\hline $\mathrm{pH}$ & & * & * \\
\hline $\mathrm{DIC}\left(\mathrm{mg} \mathrm{l}^{-1}\right)$ & & $*$ & $*$ \\
\hline $\operatorname{DOC}\left(\mathrm{mg} \mathrm{l}^{-1}\right)$ & & & $*$ \\
\hline $\operatorname{COND}\left(\mu \mathrm{S} \mathrm{cm}^{-1}\right)$ & & $*$ & $*$ \\
\hline $\mathrm{Cl}\left(\mathrm{mg} \mathrm{l}^{-1}\right)$ & & $*$ & $*$ \\
\hline $\mathrm{Ca}\left(\mathrm{mg} \mathrm{l}^{-1}\right)$ & $*$ & $*$ & $*$ \\
\hline $\mathrm{K}\left(\mathrm{mg} \mathrm{l}^{-1}\right)$ & $*$ & $*$ & $*$ \\
\hline $\operatorname{Mg}\left(\mathrm{mg} \mathrm{l}^{-1}\right)$ & & $*$ & $*$ \\
\hline $\mathrm{Na}\left(\mathrm{mg} \mathrm{l}^{-1}\right)$ & & * & $*$ \\
\hline $\mathrm{SO}_{4}\left(\mathrm{mg} \mathrm{l}^{-1}\right)$ & & * & * \\
\hline \multicolumn{4}{|l|}{$\mathrm{SiO}_{2}\left(\mathrm{mg} \mathrm{l}^{-1}\right)$} \\
\hline Chl- $a\left(\mu \mathrm{g} \mathrm{1^{-1 }}\right)$ & $*$ & & $*$ \\
\hline \multicolumn{4}{|l|}{ TP-UF $\left(\mu \mathrm{g} \mathrm{l}^{-1}\right)$} \\
\hline $\mathrm{TN}\left(\mu \mathrm{g} \mathrm{1^{-1 }}\right)$ & $*$ & $*$ & $*$ \\
\hline $\mathrm{TN}: \mathrm{TP}$ & $*$ & $*$ & $*$ \\
\hline $\mathrm{TKN}\left(\mu \mathrm{g}^{-1}\right)$ & $*$ & $*$ & $*$ \\
\hline TEMP $\left({ }^{\circ} \mathrm{C}\right)$ & & $*$ & $*$ \\
\hline \multicolumn{4}{|l|}{$\mathrm{Fe}\left(\mu \mathrm{g} 1^{-1}\right)$} \\
\hline $\mathrm{Ba}\left(\mathrm{mg} \mathrm{l}^{-1}\right)$ & & $*$ & $*$ \\
\hline $\operatorname{Mn}\left(\operatorname{mg~}^{-1}\right)$ & & $*$ & $*$ \\
\hline $\operatorname{Li}\left(\mathrm{mg} \mathrm{l}^{-1}\right)$ & & $*$ & $*$ \\
\hline $\operatorname{Sr}\left(\mathrm{mg} \mathrm{l}^{-1}\right)$ & $*$ & $*$ & $*$ \\
\hline $\mathrm{Al}\left(\mathrm{mg} \mathrm{l}^{-1}\right)$ & $*$ & $*$ & $*$ \\
\hline \multicolumn{4}{|l|}{ DEPTH (m) } \\
\hline AREA (ha) & & & \\
\hline
\end{tabular}

\subsection{Patterns in physical variables across vegetation zones}

The 56 study lakes were generally small (surface area ranged from 0.9 to 36.6 ha; median $=7.9$ ha) and shallow (maximum depth ranged from 0.5 to $19.0 \mathrm{~m}$; median $=3.5 \mathrm{~m})($ Tab. 4). There were no significant differences ( $<<0.05$; Tab. 2) and no pattern was seen in these variables across the three ecozones, as each region contained both deeper and shallower lakes, as well as lakes with both smaller and larger surface areas (Fig. 2a).

Surface lakewater temperature varied from $9.1^{\circ} \mathrm{C}$ to $22.0^{\circ} \mathrm{C}$, with a median for the full lakeset of $15.5^{\circ} \mathrm{C}$ (Tab. 4). Similar to Pienitz et al. (1997a), surface water temperature decreased from south to north along the latitudinal gradient with the warmest temperatures recorded in the boreal forest lakes (median $=17.4{ }^{\circ} \mathrm{C}$ ), and the coolest in the arctic tundra (median $=13.1^{\circ} \mathrm{C}$ ) (Fig. 2b). As surface water temperatures in shallower lakes vary considerably within a given season, and from year to year, the data presented here should be considered as a measure of relative differences among zones, as these lakes were sampled within a short time window in August but in two different years. 


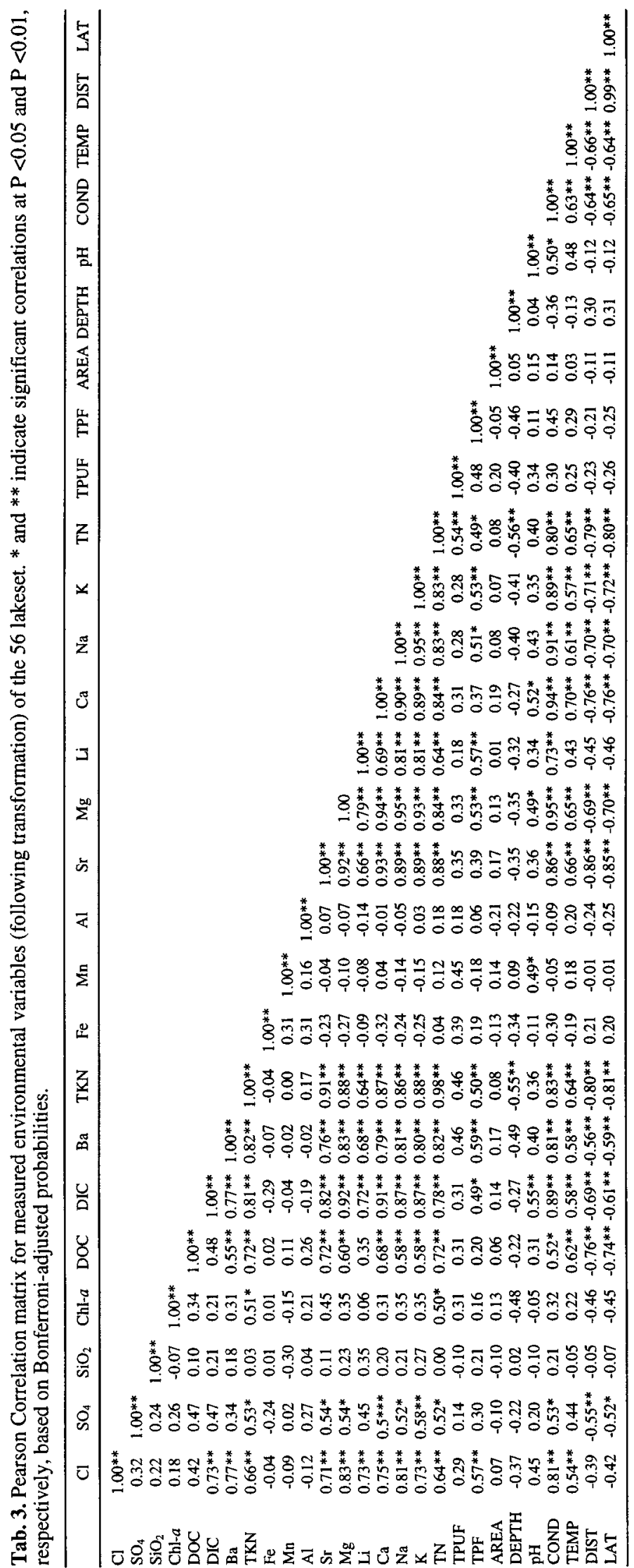


Tab. 4. Limnological measurements for the 56 study sites including minimum, maximum, mean, and median values for each ecozone. D.N.A. data not available.

\begin{tabular}{|c|c|c|c|c|c|c|c|c|c|c|c|c|c|c|c|}
\hline LAKE & $\mathrm{Cl}$ & $\mathrm{SO}_{4}$ & $\frac{\mathrm{SiO}_{2}}{\left(\mathrm{mg} \mathrm{l}^{-1}\right)}$ & $\mathrm{DOC}$ & DIC & $\begin{array}{c}\mathrm{Fe} \\
\left(\mu \mathrm{g} \mathrm{l}^{-1}\right)\end{array}$ & \multicolumn{8}{|c|}{$\left(\mathrm{mg} \mathrm{l}^{-1}\right)$} & $\mathrm{Al}$ \\
\hline \multicolumn{16}{|c|}{ Arctic Tundra } \\
\hline TK-1 & 0.82 & 0.8 & 0.51 & 2.4 & 2.9 & 57.0 & 1.30 & 0.20 & 0.9 & 0.3 & 0.7 & 0.001 & 0.001 & 0.002 & 0.020 \\
\hline TK-2 & 0.64 & 0.7 & 0.27 & 2.0 & 0.8 & 80.0 & 5.30 & 0.27 & 0.4 & 0.2 & 0.3 & 0.001 & 0.001 & 0.002 & 0.010 \\
\hline TK-6 & 0.97 & 3.5 & 0.20 & 1.7 & 1.7 & 44.0 & 1.90 & 0.20 & 0.7 & 0.2 & 0.6 & 0.001 & 0.001 & 0.002 & 0.010 \\
\hline TK-7 & 0.92 & 1.7 & 0.12 & 1.3 & 1.3 & 25.0 & 2.70 & 0.27 & 0.5 & 0.2 & 0.4 & 0.001 & 0.001 & 0.001 & 0.010 \\
\hline TK-8 & 0.60 & 0.9 & 0.66 & 5.9 & 4.7 & 89.0 & 6.50 & 0.27 & 2.5 & 0.3 & 1.8 & 0.001 & 0.002 & 0.008 & 0.010 \\
\hline TK-9 & 6.90 & 0.2 & 0.13 & 2.7 & 1.5 & 30.0 & 6.40 & 0.60 & 2.6 & 0.4 & 1.4 & 0.001 & 0.011 & 0.010 & 0.010 \\
\hline TK-10 & 0.46 & 1.1 & 0.31 & 3.0 & 1.8 & 57.0 & 1.80 & 0.27 & 1.2 & 0.2 & 0.6 & 0.001 & 0.001 & 0.005 & 0.010 \\
\hline TK-11 & 0.51 & 1.0 & 0.52 & 2.4 & 1.0 & 52.0 & 2.40 & 0.27 & 0.4 & 0.2 & 0.3 & 0.001 & 0.001 & 0.004 & 0.020 \\
\hline TK-12 & 0.32 & 4.1 & 0.52 & 1.9 & 2.2 & 39.0 & 1.50 & 0.27 & 1.0 & 0.5 & 0.8 & 0.003 & 0.002 & 0.001 & 0.010 \\
\hline TK-13 & 0.28 & 5.8 & 2.47 & 2.3 & 1.2 & 23.0 & 3.70 & 0.20 & 1.0 & 0.5 & 0.8 & 0.003 & 0.002 & 0.002 & 0.050 \\
\hline TK-14 & 0.21 & 2.5 & 0.95 & 1.7 & 0.9 & 37.0 & 3.60 & 0.27 & 0.5 & 0.3 & 0.3 & 0.001 & 0.001 & 0.002 & 0.040 \\
\hline TK-15 & 0.23 & 2.4 & 1.21 & 1.3 & 1.2 & 21.0 & 1.40 & 0.27 & 0.5 & 0.4 & 0.3 & 0.001 & 0.001 & 0.002 & 0.040 \\
\hline TK-16 & 0.19 & 1.7 & 0.26 & 1.3 & 0.7 & 9.0 & 1.30 & 0.27 & 0.3 & 0.3 & 0.2 & 0.001 & 0.001 & 0.002 & 0.010 \\
\hline TK-17 & 0.21 & 2.2 & 0.33 & 1.7 & 0.9 & 9.0 & 1.60 & 0.27 & 0.5 & 0.4 & 0.3 & 0.001 & 0.001 & 0.002 & 0.020 \\
\hline TK-18 & 0.21 & 0.8 & 0.46 & 3.3 & 1.8 & 253.0 & 2.90 & 0.27 & 0.6 & 0.4 & 0.4 & 0.001 & 0.001 & 0.006 & 0.010 \\
\hline TK-19 & 0.36 & 1.2 & 0.29 & 5.3 & 2.3 & 418.0 & 2.30 & 0.27 & 0.7 & 0.4 & 0.6 & 0.001 & 0.003 & 0.009 & 0.020 \\
\hline TK-20 & 0.16 & 1.7 & 0.26 & 2.3 & 1.2 & 23.0 & 4.10 & 0.50 & 0.5 & 0.3 & 0.3 & 0.001 & 0.001 & 0.001 & 0.010 \\
\hline TK-21 & 0.81 & 1.9 & 0.25 & 10.3 & 2.9 & 350.0 & 4.40 & 0.50 & 1.4 & 0.6 & 1.1 & 0.002 & 0.008 & 0.007 & 0.001 \\
\hline Min. & 0.16 & 0.2 & 0.12 & 1.3 & 0.7 & 9.0 & 1.30 & 0.20 & 0.3 & 0.2 & 0.2 & 0.001 & 0.001 & 0.001 & 0.001 \\
\hline Max. & 6.90 & 5.8 & 2 & 10.3 & 4.7 & 418.0 & 6. & 0.60 & 2.6 & 0.6 & 1.8 & 0.003 & 0.011 & 0.010 & 0.050 \\
\hline Mean & 0.82 & 1.9 & 0.54 & 2.9 & 1.7 & 89.8 & 3.06 & 0.60 & 0.9 & 0.3 & 0.6 & 0.001 & 0.002 & 0.004 & 0.017 \\
\hline Median & 0.41 & 1.7 & 0.32 & 2.3 & 1.4 & 41.5 & 2.55 & 0.27 & 0.7 & 0.3 & 0.5 & 0.001 & 0.001 & 0.002 & 0.010 \\
\hline \multicolumn{16}{|c|}{ Forest-Tundra } \\
\hline TK-29 & 0.32 & 1.1 & 0.5 & 138. & 1.8 & & 6 & 0. & 1.50 & 0.40 & 0.60 & 0.001 & 0.004 & 0.003 & 0.010 \\
\hline TK-30 & 0.81 & 3.5 & 0.56 & 30.4 & 0.1 & 531.0 & 8.00 & 0.60 & 1.20 & 0.60 & 0.70 & 0.002 & 0.005 & 0.004 & 0.260 \\
\hline TK-31 & 0.85 & 2.8 & 0.89 & 27.3 & 1.6 & 623.0 & 7.60 & 0.30 & 1.30 & 0.40 & 0.50 & 0.001 & 0.004 & 0.004 & 0.130 \\
\hline TK-32 & 0.71 & 2.7 & 0.15 & 17.5 & 3.2 & 437.0 & 5.70 & 0.30 & 1.20 & 0.40 & 0.60 & 0.001 & 0.006 & 0.003 & 0.130 \\
\hline TK-50 & 0.49 & 1.5 & 0.70 & 13.4 & 2.4 & 51.0 & 3.10 & 0.40 & 2.80 & 0.60 & 1.10 & 0.001 & 0.010 & 0.002 & 0.030 \\
\hline TK-51 & 0.39 & 1.7 & 0.34 & 11.0 & 1.3 & 42.0 & 3.10 & 0.50 & 1.30 & 0.70 & 0.80 & 0.001 & 0.009 & 0.003 & 0.070 \\
\hline TK-52 & 0.56 & 1.9 & 0.17 & 14.0 & 1.0 & 38.0 & 0.70 & 0.50 & 1.00 & 0.60 & 0.80 & 0.001 & 0.005 & 0.002 & 0.070 \\
\hline TK-53 & 0.44 & 2.4 & 2.04 & 13.4 & 1.7 & 52.0 & 1.00 & 0.20 & 2.50 & 0.60 & 1.10 & 0.001 & 0.007 & 0.003 & 0.110 \\
\hline TK-54 & 0.19 & 3.2 & 0.14 & 5.8 & 0.7 & 4.0 & 2.70 & 0.27 & 1.00 & 0.40 & 0.50 & 0.001 & 0.005 & 0.002 & 0.010 \\
\hline TK-55 & 0.27 & 1.5 & 0.18 & 10.4 & 2.8 & 33.0 & 3.10 & 0.80 & 1.80 & 0.90 & 1.00 & 0.001 & 0.009 & 0.003 & 0.020 \\
\hline TK-56 & 0.34 & 1.7 & 0.72 & 11.4 & 2.5 & 32.0 & 0.70 & 1.20 & 2.80 & 0.80 & 1.10 & 0.001 & 0.009 & 0.002 & 0.020 \\
\hline Min. & 0.19 & 1.1 & 0.14 & 5.8 & 0.1 & 4 & 0.70 & 0.20 & 1.00 & 0.40 & 0.50 & 0.001 & 0.004 & 0.002 & 0.01 \\
\hline tiva & 0.85 & 3.5 & 2.04 & 138.0 & 3.2 & 623.0 & 8.00 & 1.20 & 2.80 & 0.90 & 1.1 & 0.002 & 0.010 & 0.004 & 0.26 \\
\hline Mean & 0.49 & 2.2 & 0.58 & 26.6 & 1. & 169 & 3.85 & 0.48 & 1.67 & 0.58 & 0.80 & 0.001 & 0.007 & 0.003 & 0.08 \\
\hline Median & 0.44 & 1.9 & 0.53 & 13.4 & 1.7 & 42.0 & 3.10 & 0.40 & 1.30 & 0.60 & 0.80 & 0.001 & 0.006 & 0.003 & 0.07 \\
\hline
\end{tabular}


Tab. 4. Continued

\begin{tabular}{|c|c|c|c|c|c|c|c|c|c|c|c|c|c|c|}
\hline \multirow[t]{2}{*}{ LAKE } & $\mathrm{TN}$ & TPUF & TKN & CHL $a$ & $\mathrm{POC}$ & $\mathrm{PON}$ & \multirow[t]{2}{*}{ TN:TP } & \multirow{2}{*}{ POC:CHL $a$} & \multirow{2}{*}{$\begin{array}{c}\text { AREA } \\
\text { (ha) }\end{array}$} & \multirow{2}{*}{$\begin{array}{l}\text { DEPTH } \\
(\mathrm{m})\end{array}$} & \multirow[t]{2}{*}{$\mathrm{pH}$} & \multirow{2}{*}{$\begin{array}{c}\text { COND } \\
\left(\mu \mathrm{S} \mathrm{cm}^{-1}\right)\end{array}$} & \multirow{2}{*}{$\begin{array}{c}\text { TEMP } \\
\left({ }^{\circ} \mathrm{C}\right)\end{array}$} & \multirow{2}{*}{$\begin{array}{l}\text { DIST } \\
(\mathrm{km})\end{array}$} \\
\hline & & & \multicolumn{2}{|c|}{$\left(\mu \mathrm{g} \mathrm{l}^{-1}\right)$} & & & & & & & & & & \\
\hline \multicolumn{15}{|c|}{ Arctic Tundra } \\
\hline TK-1 & 208.0 & 27.1 & 161.0 & 0.4 & 330.0 & 28.0 & 7.7 & 825.0 & 5.9 & 9.5 & 7.7 & 11.7 & 17.3 & 285.0 \\
\hline TK-2 & 135.0 & 3.1 & 93.0 & 1.0 & 313.0 & 32.0 & 43.5 & 313.0 & 2.8 & 5.5 & 8.0 & 9.8 & 12.9 & 265.0 \\
\hline TK-6 & 159.0 & 4.2 & 130.0 & 1.2 & 204.0 & 19.0 & 37.9 & 170.0 & 5.3 & 4.5 & 8.0 & 17.0 & 12.1 & 320.0 \\
\hline TK-7 & 165.0 & 8.4 & 125.0 & 1.4 & 322.0 & 30.0 & 19.6 & 230.0 & 1.8 & 9.5 & 7.6 & 12.3 & 12.4 & 310.0 \\
\hline TK-8 & 451.0 & 20.1 & 323.0 & 2.2 & 1190.0 & 118.0 & 22.4 & 540.9 & 8.5 & 3.0 & 7.7 & 98.8 & 14.7 & 115.0 \\
\hline TK-9 & 228.0 & 13.4 & 175.0 & 1.1 & 362.0 & 43.0 & 17.0 & 329.1 & 35.7 & 5.5 & 7.5 & 36.3 & 14.3 & 140.0 \\
\hline TK-10 & 234.0 & 5.3 & 181.0 & 1.5 & 454.0 & 43.0 & 44.2 & 302.7 & 36.6 & 3.0 & 7.6 & 14.8 & 14.0 & 185.0 \\
\hline TK-11 & 185.0 & 5.6 & 124.0 & 0.6 & 527.0 & 51.0 & 33.0 & 878.3 & 6.3 & 3.5 & 6.8 & 9.7 & 13.9 & 192.5 \\
\hline TK-12 & 198.0 & 7.4 & 146.0 & 0.7 & 420.0 & 42.0 & 26.8 & 600.0 & 13.1 & 6.1 & 6.9 & 13.9 & 10.4 & 175.0 \\
\hline TK-13 & 198.0 & 5.9 & 155.0 & 0.4 & 210.0 & 25.0 & 33.6 & 525.0 & 2.6 & 3.1 & 6.9 & 16.1 & 13.0 & 85.0 \\
\hline TK-14 & 170.0 & 4.3 & 121.0 & 1.4 & 301.0 & 32.0 & 39.5 & 215.0 & 2.8 & 4.9 & 6.4 & 9.2 & 13.1 & 100.0 \\
\hline TK-15 & 146.0 & 4.6 & 99.0 & 0.7 & 245.0 & 23.0 & 31.7 & 350.0 & 0.9 & 3.0 & 6.6 & 9.4 & 12.4 & 97.5 \\
\hline TK-16 & 150.0 & 4.2 & 106.0 & 0.7 & 272.0 & 32.0 & 35.7 & 388.6 & 4.2 & 6.0 & 6.6 & 7.3 & 13.7 & 85.0 \\
\hline TK-17 & 137.0 & 2.7 & 108.0 & 0.7 & 190.0 & 19.0 & 50.7 & 271.4 & 10.3 & 5.9 & 6.6 & 9.3 & 14.0 & 95.0 \\
\hline TK-18 & 258.0 & 31.3 & 180.0 & 1.1 & 665.0 & 68.0 & 8.2 & 604.5 & 10.6 & 7.3 & 6.6 & 8.7 & 11.3 & 55.0 \\
\hline TK-19 & 566.0 & 28.1 & 499.0 & 0.7 & 516.0 & 56.0 & 20.1 & 737.1 & 8.4 & 0.8 & 6.7 & 9.7 & 9.1 & 55.0 \\
\hline TK-20 & 186.0 & 6.2 & 145.0 & 0.7 & 337.0 & 31.0 & 30.0 & 481.4 & 9.7 & 8.8 & 7.2 & 8.7 & 12.8 & 35.0 \\
\hline TK-21 & 793.0 & 28.3 & 527.0 & 6.7 & 2990.0 & 256.0 & 28.0 & 446.3 & 9.7 & 1.0 & 7.0 & 17.1 & 13.5 & 23.0 \\
\hline Min. & 135.0 & 2.7 & 93.0 & 0.4 & 190.0 & 19.0 & 7.7 & 170.0 & 0.9 & 0.8 & 6.4 & 7.3 & 9.1 & 23.0 \\
\hline Max. & 793.0 & 31.3 & 527.0 & 6.7 & 2990.0 & 256.0 & 50.7 & 878.3 & 36.6 & 9.5 & 8.0 & 98.8 & 17.3 & 320.0 \\
\hline Mean & 253.7 & 11.7 & 188.8 & 1.3 & 547.1 & 52.7 & 29.4 & 456.0 & 9.7 & 5.0 & 7.1 & 17.8 & 13.1 & 145.4 \\
\hline Median & 192.0 & 6.1 & 145.5 & 0.9 & 333.5 & 32.0 & 30.9 & 417.4 & 7.4 & 5.2 & 7.0 & 10.8 & 13.1 & 107.5 \\
\hline \multicolumn{15}{|c|}{ Forest-Tundra } \\
\hline TK-29 & 287.0 & 4.20 & 203.0 & 0.7 & 710.0 & 74.0 & 68.3 & 1014.3 & 18.0 & 10.1 & 7.7 & 14.6 & 16.1 & -65.0 \\
\hline TK-30 & 593.0 & 28.50 & 352.0 & 4.6 & 3250.0 & 231.0 & 20.8 & 706.5 & 10.7 & 1.5 & 7.3 & 15.0 & 15.8 & -45.0 \\
\hline TK-31 & 403.0 & 9.20 & 312.0 & 1.8 & 805.0 & 81.0 & 43.8 & 447.2 & 3.5 & 5.0 & 6.8 & 13.4 & 16.8 & -35.0 \\
\hline TK-32 & 614.0 & 24.50 & 449.0 & 2.1 & 1830.0 & 155.0 & 25.1 & 871.4 & 5.9 & 1.5 & 7.5 & 13.7 & 16.3 & -50.0 \\
\hline TK-50 & 410.0 & 4.50 & 423.0 & 4.3 & D.N.A. & D.N.A. & 91.1 & 0.0 & 4.3 & 1.8 & 6.8 & 26.2 & 12.5 & -50.0 \\
\hline TK-51 & 373.0 & 9.40 & 357.0 & 7.9 & D.N.A. & D.N.A. & 39.7 & 0.0 & 8.8 & 1.8 & 6.4 & 18.4 & 12.0 & -22.5 \\
\hline TK-52 & 413.0 & 6.00 & 444.0 & 4.3 & D.N.A. & D.N.A. & 68.8 & 0.0 & 9.0 & 1.9 & 6.2 & 18.1 & 12.2 & -25.0 \\
\hline TK-53 & 311.0 & 4.20 & 317.0 & 1.8 & D.N.A. & D.N.A. & 74.0 & 0.0 & 4.6 & 19.0 & 6.6 & 19.8 & 14.4 & -30.0 \\
\hline TK-54 & 166.0 & 3.10 & 167.0 & 1.8 & D.N.A. & D.N.A. & 53.5 & 0.0 & 9.9 & 3.4 & 6.2 & 12.3 & 13.0 & -10.0 \\
\hline TK-55 & 395.0 & 5.30 & 422.0 & 3.9 & D.N.A. & D.N.A. & 74.5 & 0.0 & 9.8 & 2.6 & 7.0 & 20.6 & 13.7 & -40.0 \\
\hline TK-56 & 298.0 & 7.80 & 324.0 & 3.8 & D.N.A. & D.N.A. & 38.2 & 0.0 & 13.2 & 6.0 & 6.9 & 25.2 & 14.5 & -75.0 \\
\hline Min. & 166.0 & 3.10 & 167.0 & 0.7 & 710.0 & 74.0 & 20.8 & 447.2 & 3.5 & 1.5 & 6.2 & 12.3 & 12.0 & -75.0 \\
\hline Max. & 614.0 & 28.50 & 449.0 & 7.9 & 3250.0 & 231.0 & 91.1 & 1014.3 & 18.0 & 19.0 & 7.7 & 26.2 & 16.8 & -10.0 \\
\hline Mean & 387.5 & 9.70 & 342.7 & 3.4 & 1648.8 & 135.3 & 54.4 & 759.9 & 8.9 & 5.0 & 6.8 & 17.9 & 14.3 & -40.7 \\
\hline Median & 395.0 & 6.00 & 352.0 & 3.8 & 1317.5 & 118.0 & 53.5 & 789.0 & 9.0 & 2.6 & 6.8 & 18.1 & 14.4 & -40.0 \\
\hline
\end{tabular}


Tab. 4. Continued

\begin{tabular}{|c|c|c|c|c|c|c|c|c|c|c|c|c|c|c|c|}
\hline LAKE & $\mathrm{Cl}$ & $\mathrm{SO}_{4}$ & $\frac{\mathrm{SiO}_{2}}{\left(\mathrm{mg} \mathrm{l}^{-1}\right)}$ & $\mathrm{DOC}$ & DIC & $\begin{array}{c}\mathrm{Fe} \\
\left(\mu \mathrm{g} \mathrm{l}^{-1}\right)\end{array}$ & \multicolumn{9}{|c|}{$\left(\mathrm{mg} \mathrm{l}^{-1}\right)$} \\
\hline \multicolumn{16}{|c|}{ Boreal Forest } \\
\hline TK-22 & 2.0 & 4.1 & 0.7 & 12.8 & 6.9 & 50.0 & 10.30 & 1.50 & 8.1 & 0.9 & 2.9 & 0.004 & 0.037 & 0.008 & 0.020 \\
\hline TK-23 & 0.4 & 1.6 & 0.1 & 15.2 & 2.8 & 84.0 & 6.60 & 0.40 & 3.4 & 0.4 & 0.9 & 0.001 & 0.009 & 0.002 & 0.040 \\
\hline TK-24 & 1.1 & 8.6 & 0.2 & 24.3 & 9.9 & 12.0 & 52.60 & 1.80 & 17.9 & 1.6 & 3.8 & 0.003 & 0.044 & 0.011 & 0.030 \\
\hline TK-25 & 0.7 & 1.5 & 0.1 & 16.5 & 7.3 & 40.0 & 49.50 & 1.20 & 8.1 & 1.2 & 2.0 & 0.003 & 0.025 & 0.005 & 0.010 \\
\hline TK-26 & 0.6 & 3.9 & 0.1 & 6.2 & 7.3 & 4.0 & 7.30 & 1.10 & 7.5 & 1.2 & 2.5 & 0.002 & 0.023 & 0.002 & 0.010 \\
\hline TK-27 & 1.1 & 4.9 & 0.5 & 11.0 & 7.5 & 31.0 & 43.40 & 1.50 & 7.1 & 1.1 & 3.8 & 0.003 & 0.022 & 0.004 & 0.030 \\
\hline TK-28 & 0.7 & 7.5 & 1.4 & 165.0 & 9.0 & 22.0 & 6.90 & 0.60 & 15.6 & 1.0 & 2.1 & 0.001 & 0.019 & 0.005 & 0.020 \\
\hline TK-33 & 0.5 & 1.6 & 0.4 & 116.0 & 2.3 & 34.0 & 3.10 & 0.40 & 2.4 & 0.3 & 1.0 & 0.001 & 0.006 & 0.003 & 0.040 \\
\hline TK-34 & 0.4 & 0.7 & 0.2 & 15.0 & 2.7 & 26.0 & 3.00 & 0.50 & 3.3 & 0.5 & 1.0 & 0.001 & 0.009 & 0.005 & 0.050 \\
\hline TK-35 & 0.6 & 6.3 & 0.3 & 332.0 & 1.4 & 21.0 & 4.70 & 0.80 & 6.3 & 0.6 & 1.6 & 0.001 & 0.019 & 0.005 & 0.020 \\
\hline TK-36 & 1.7 & 7.0 & 0.3 & 80.1 & 2.1 & 549.0 & 16.5 & 1.50 & 4.9 & 1.0 & 2.1 & 0.003 & 0.015 & 0.006 & 0.130 \\
\hline TK-37 & 41.1 & 3.4 & 1.4 & 65.1 & 41.0 & 205.0 & 9.30 & 39.80 & 30.7 & 14.8 & 32.4 & 0.028 & 0.197 & 0.060 & 0.010 \\
\hline TK-38 & 0.9 & 3.2 & 0.7 & 26.5 & 6.4 & 21.0 & 9.30 & 0.90 & 7.7 & 1.4 & 2.7 & 0.004 & 0.021 & 0.009 & 0.030 \\
\hline TK-39 & 15.4 & 5.8 & 0.4 & 69.9 & 26.1 & 27.0 & 22.60 & 5.40 & 34.7 & 6.2 & 14.7 & 0.005 & 0.123 & 0.061 & 0.050 \\
\hline TK-40 & 33.4 & 6.1 & 0.9 & 30.2 & 13.8 & 13.0 & 9.30 & 5.50 & 21.1 & 2.1 & 12.6 & 0.005 & 0.080 & 0.015 & 0.010 \\
\hline TK-41 & 1.8 & 13.3 & 0.3 & 51.6 & 11.2 & 52.0 & 2.70 & 4.10 & 19.3 & 4.2 & 5.0 & 0.003 & 0.058 & 0.030 & 0.210 \\
\hline TK-42 & 1.8 & 10.5 & 0.1 & 44.8 & 12.2 & 24.0 & 4.70 & 3.20 & 10.8 & 3.5 & 10.5 & 0.001 & 0.047 & 0.017 & 0.060 \\
\hline TK-43 & 0.5 & 2.4 & 0.1 & 13.4 & 3.1 & 4.0 & 1.4 & 0.70 & 3.4 & 0.6 & 2.0 & 0.001 & 0.012 & 0.004 & 0.010 \\
\hline TK-44 & 16.6 & 2.6 & 0.2 & 26.2 & 8.0 & 21.0 & 9.30 & 2.40 & 10.2 & 1.9 & 7.5 & 0.003 & 0.048 & 0.014 & 0.010 \\
\hline TK-45 & 7.3 & 10.3 & 0.7 & 24.8 & 21.7 & 2.0 & 9.3 & 7.50 & 20.4 & 3.8 & 13.6 & 0.007 & 0.101 & 0.025 & 0.010 \\
\hline TK-46 & 13.0 & 3.2 & 2.3 & 61.1 & 42.3 & 46.0 & 9.3 & 31.30 & 29.3 & 16.8 & 32.2 & 0.022 & 0.129 & 0.027 & 0.010 \\
\hline TK-47 & 0.4 & 2.2 & 0.2 & 17.4 & 2.6 & 5.0 & 9.30 & 0.50 & 3.8 & 0.6 & 1.2 & 0.001 & 0.012 & 0.009 & 0.030 \\
\hline TK-48 & 0.9 & 2.7 & 0.2 & 24.6 & 10.1 & 14.0 & 12.10 & 1.80 & 15.0 & 2.0 & 2.7 & 0.001 & 0.039 & 0.007 & 0.030 \\
\hline TK-4 & 2.7 & 5.9 & 5.7 & 9. & 9.0 & $9 .($ & 9.30 & 2.00 & 12.5 & 1.2 & 3.0 & 0.001 & 0.023 & 0.004 & 0.010 \\
\hline TK-57 & 0.7 & 4.5 & 0.9 & 16.4 & 2.1 & 59.0 & 9.30 & 0.70 & 2.6 & 1.0 & 1.7 & 0.001 & 0.014 & 0.003 & 0.060 \\
\hline TK-58 & 1.0 & 5.1 & 0.2 & 26.2 & 9.1 & 5.0 & 9.30 & 4.00 & 5.6 & 2.5 & 5.2 & 0.002 & 0.035 & 0.008 & 0.020 \\
\hline TK-59 & 3.5 & 4.7 & 3.4 & 27.7 & 35.4 & 1.0 & 9.30 & 12.20 & 37.0 & 9.3 & 23.2 & 0.010 & 0.130 & 0.034 & 0.010 \\
\hline Min. & 0.4 & 0.7 & 0.1 & 6. & 1. & 1. & 1.40 & 0.4 & 2. & 0.3 & 0. & 0.001 & 0.006 & 0.002 & 0.010 \\
\hline Max. & 41.1 & 13.3 & 5.7 & 332.0 & 42.3 & 549.0 & 52.60 & 39.80 & 37.0 & 16.8 & 32.4 & 0.028 & 0.197 & 0.061 & 0.210 \\
\hline Mean & 5.6 & 4.9 & 0.8 & 49.2 & 11.6 & 51.1 & 12.95 & 4.94 & 12.9 & 3.0 & 7.2 & 0.004 & 0.048 & 0.014 & 0.036 \\
\hline Median & 1.1 & 4.5 & 0.3 & 26.2 & 8.0 & 22.0 & 9.30 & 1.50 & 8.1 & 1.2 & 2.9 & 0.003 & 0.025 & 0.008 & 0.020 \\
\hline \multicolumn{16}{|c|}{ Full Lakeset (56 lakes) } \\
\hline Min. & 0 & 0 & 0.0 & & 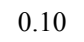 & & 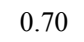 & 0.2 & 0. & 0.2 & 0.2 & 0.001 & 0.001 & 0.001 & 0.001 \\
\hline Max. & 41.10 & 13.30 & 5.72 & 332.00 & 42.30 & 623.00 & 52.60 & 39.80 & 37.00 & 16.80 & 32.40 & 0.028 & 0.197 & 0.061 & 0.260 \\
\hline Mean & 3.05 & 3.43 & 0.67 & 29.91 & 6.49 & 86.80 & 7.99 & 2.57 & 6.84 & 1.68 & 3.82 & 0.003 & 0.025 & 0.009 & 0.038 \\
\hline Median & 0.68 & 2.55 & 0.34 & 13.40 & 2.55 & 32.50 & 0.70 & 0.20 & 2.60 & 0.60 & 1.10 & 0.001 & 0.009 & 0.004 & 0.020 \\
\hline
\end{tabular}


Tab. 4. Continued

\begin{tabular}{|c|c|c|c|c|c|c|c|c|c|c|c|c|c|c|}
\hline \multirow[t]{2}{*}{ LAKE } & $\mathrm{TN}$ & TPUF & TKN & CHL $a$ & POC & PON & \multirow[t]{2}{*}{ TN:TP } & \multirow[t]{2}{*}{ POC:CHL $a$} & \multirow{2}{*}{$\begin{array}{l}\text { AREA } \\
\text { (ha) }\end{array}$} & \multirow{2}{*}{$\begin{array}{l}\text { DEPTH } \\
(\mathrm{m})\end{array}$} & \multirow[t]{2}{*}{$\mathrm{pH}$} & \multirow{2}{*}{$\begin{array}{c}\text { COND } \\
\left(\mu \mathrm{S} \mathrm{cm}^{-1}\right)\end{array}$} & \multirow{2}{*}{$\begin{array}{l}\text { TEMP } \\
\left({ }^{\circ} \mathrm{C}\right)\end{array}$} & \multirow{2}{*}{$\begin{array}{l}\text { DIST } \\
(\mathrm{km})\end{array}$} \\
\hline & \multicolumn{6}{|c|}{$\left(\mu \mathrm{g}^{-1}\right)$} & & & & & & & & \\
\hline \multicolumn{15}{|c|}{ Boreal Forest } \\
\hline TK-22 & 660.0 & 16.2 & 472.0 & 3.5 & 2050.0 & 178.0 & 40.7 & 585.7 & 8.8 & 2.8 & 7.4 & 62.0 & 17.5 & -160.0 \\
\hline TK-23 & 633.0 & 7.9 & 553.0 & 1.9 & 815.0 & 70.0 & 80.1 & 428.9 & 6.9 & 2.0 & 7.6 & 24.2 & 17.4 & -155.0 \\
\hline TK-24 & 1392.0 & 11.0 & 1170.0 & 1.1 & 3270.0 & 212.0 & 126.5 & 2972.7 & 16.8 & 8.0 & 8.2 & 102.2 & 17.1 & -135.0 \\
\hline TK-25 & 966.0 & 10.0 & 810.0 & 1.8 & 1910.0 & 146.0 & 96.6 & 1061.1 & 2.7 & 6.0 & 7.9 & 53.9 & 17.4 & -100.0 \\
\hline TK-26 & 325.0 & 6.9 & 275.0 & 0.6 & 350.0 & 40.0 & 47.1 & 583.3 & 20.9 & 16.0 & 7.7 & 56.9 & 16.9 & -130.0 \\
\hline TK-27 & 672.0 & 23.0 & 501.0 & 2.0 & 1860.0 & 161.0 & 29.2 & 930.0 & 10.1 & 3.0 & 8.0 & 64.6 & 17.8 & -170.0 \\
\hline TK-28 & 512.0 & 10.8 & 441.0 & 1.2 & 690.0 & 61.0 & 47.4 & 575.0 & 8.8 & 6.1 & 7.8 & 74.8 & 16.5 & -110.0 \\
\hline TK-33 & 363.0 & 6.1 & 306.0 & 1.1 & 426.0 & 47.0 & 59.5 & 387.3 & 6.1 & 10.0 & 7.3 & 2.5 & 16.3 & -80.0 \\
\hline TK-34 & 448.0 & 12.1 & 355.0 & 1.3 & 1010.0 & 83.0 & 37.0 & 776.9 & 3.6 & 5.0 & 7.4 & 25.9 & 17.4 & -95.0 \\
\hline TK-35 & 516.0 & 15.6 & 433.0 & 1.2 & 679.0 & 64.0 & 33.1 & 565.8 & 4.3 & 7.0 & 7.5 & 44.1 & 17.2 & -135.0 \\
\hline TK-36 & 996.0 & 23.3 & 881.0 & 4.8 & 960.0 & 102.0 & 42.7 & 200.0 & 4.2 & 1.5 & 7.2 & 39.1 & 17.9 & -162.5 \\
\hline TK-37 & 1790.0 & 6.4 & 2460.0 & 1.3 & D.N.A. & D.N.A. & 279.7 & D.N.A. & 6.7 & 1.3 & 7.6 & 478.5 & 18.3 & -180.0 \\
\hline TK-38 & 883.0 & 8.7 & 923.0 & 5.2 & D.N.A. & D.N.A. & 101.5 & D.N.A. & 17.4 & 2.1 & 6.3 & 64.4 & 18.2 & -170.0 \\
\hline TK-39 & 1590.0 & 48.6 & 2270.0 & 3.1 & D.N.A. & D.N.A. & 32.7 & D.N.A. & 14.4 & 1.0 & 8.3 & 271.1 & 20.2 & -155.0 \\
\hline TK-40 & 889.0 & 11.2 & 934.0 & 1.0 & D.N.A. & D.N.A. & 79.4 & D.N.A. & 7.9 & 2.2 & 7.0 & 238.9 & 18.3 & -175.0 \\
\hline TK-41 & 1930.0 & 13.0 & 2120.0 & 1.5 & D.N.A. & D.N.A. & 148.5 & D.N.A. & 4.3 & 0.5 & 7.7 & 149.5 & 22.0 & -180.0 \\
\hline TK-42 & 1590.0 & 34.8 & 1830.0 & 17.3 & D.N.A. & D.N.A. & 45.7 & D.N.A. & 8.4 & 0.9 & 7.9 & 138.5 & 19.8 & -162.5 \\
\hline TK-43 & 446.0 & 3.7 & 461.0 & 1.5 & D.N.A. & D.N.A. & 120.5 & D.N.A. & 7.3 & 7.3 & 7.1 & 42.8 & 21.5 & -160.0 \\
\hline TK-44 & 883.0 & 5.7 & 945.0 & 2.9 & D.N.A. & D.N.A. & 154.9 & D.N.A. & 2.9 & 1.4 & 7.1 & 134.0 & 20.2 & -160.0 \\
\hline TK-45 & 811.0 & 8.7 & 869.0 & 2.9 & D.N.A. & D.N.A. & 93.2 & D.N.A. & 8.0 & 6.5 & 7.8 & 228.9 & 16.8 & -150.0 \\
\hline TK-46 & 1960.0 & 10.9 & 2760.0 & 1.0 & D.N.A. & D.N.A. & 179.8 & D.N.A. & 2.6 & 0.7 & 7.8 & 405.1 & 15.7 & -152.5 \\
\hline TK-47 & 637.0 & 8.5 & 670.0 & 5.7 & D.N.A. & D.N.A. & 74.9 & D.N.A. & 4.6 & 2.5 & 6.9 & 32.8 & 14.5 & -150.0 \\
\hline TK-48 & 1020.0 & 22.1 & 1150.0 & 3.1 & D.N.A. & D.N.A. & 46.2 & D.N.A. & 18.5 & 1.5 & 7.4 & 100.2 & 13.7 & -127.5 \\
\hline TK-49 & 380.0 & 5.5 & 380.0 & 3.0 & D.N.A. & D.N.A. & 69.1 & D.N.A. & 12.2 & 3.7 & 7.4 & 97.4 & 14.3 & -100.0 \\
\hline TK-57 & 424.0 & 6.0 & 428.0 & 2.8 & D.N.A. & D.N.A. & 70.7 & D.N.A. & 3.0 & 4.6 & 6.6 & 27.6 & 15.2 & -107.5 \\
\hline TK-58 & 1080.0 & 10.8 & 1200.0 & 3.8 & D.N.A. & D.N.A. & 100.0 & D.N.A. & 2.6 & 3.2 & 7.3 & 76.9 & 16.2 & -145.0 \\
\hline TK-59 & 1030.0 & 10.6 & 1100.0 & 5.3 & D.N.A. & D.N.A. & 97.2 & D.N.A. & 15.6 & 4.6 & 8.1 & 306.5 & 19.0 & -160.0 \\
\hline Min. & 325.0 & 3.7 & 275.0 & 0.6 & 350.0 & 40.0 & 29.2 & 200.0 & 2.6 & 0.5 & 6.3 & 2.5 & 13.7 & -180.0 \\
\hline Max. & 1960.0 & 48.6 & 2760.0 & 17.3 & 3270.0 & 212.0 & 279.7 & 2972.7 & 20.9 & 16.0 & 8.3 & 478.5 & 22.0 & -80.0 \\
\hline Mean & 919.5 & 13.3 & 988.8 & 3.0 & 1274.5 & 105.8 & 86.4 & 824.3 & 8.5 & 4.1 & 7.5 & 123.8 & 17.5 & -143.2 \\
\hline Median & 883.0 & 10.8 & 869.0 & 2.0 & 960.0 & 83.0 & 74.9 & 583.3 & 7.3 & 3.0 & 7.5 & 74.8 & 17.4 & -152.5 \\
\hline \multicolumn{15}{|c|}{ Full Lakeset (56 lakes) } \\
\hline M & 135. & 2.7 & 93.0 & 0.4 & 190.0 & 19.0 & 7.7 & 170. & 0.9 & 0.5 & 6.2 & 2.5 & 9.1 & -180.0 \\
\hline Max. & 1960.0 & 48.6 & 2760.0 & 17.3 & 3270.0 & 256.0 & 279.7 & 2972.7 & 36.6 & 19.0 & 8.3 & 478.5 & 22.0 & 320.0 \\
\hline Mean & 601.0 & 12.1 & 604.7 & 2.5 & 923.1 & 80.4 & 61.8 & 615.6 & 9.0 & 4.6 & 7.2 & 68.9 & 15.5 & -30.3 \\
\hline Median & 435.0 & 8.6 & 422.5 & 1.7 & 527.0 & 56.0 & 44.9 & 540.9 & 7.9 & 3.5 & 7.3 & 25.6 & 15.5 & -70.0 \\
\hline
\end{tabular}


a)

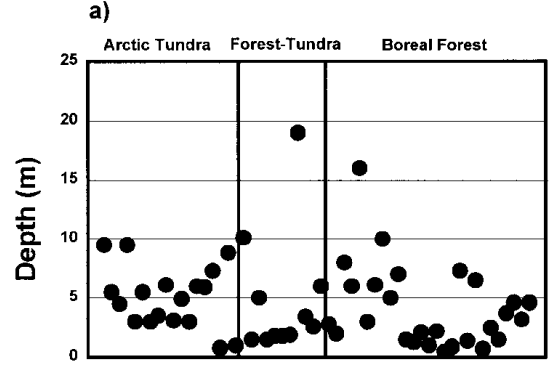

d)

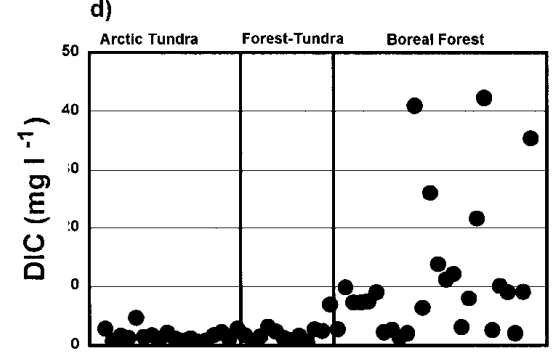

b)

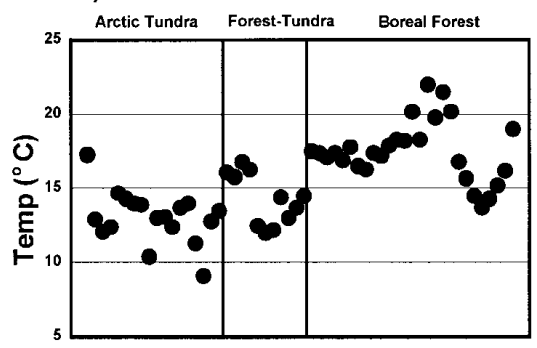

e)

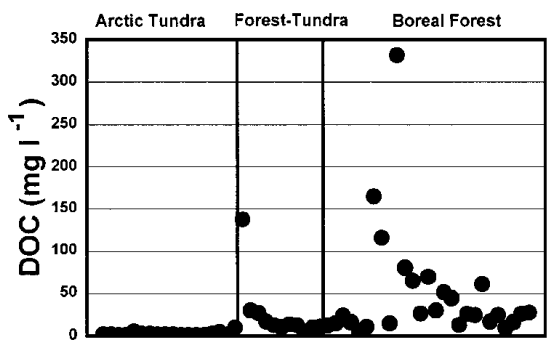

c)

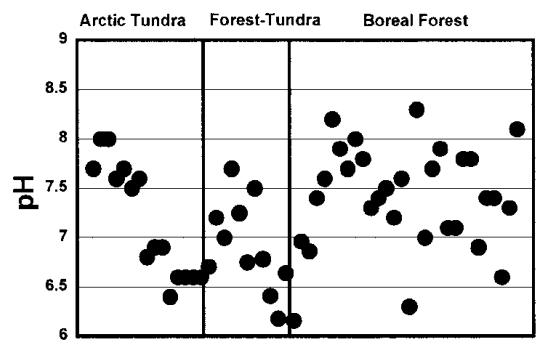

f)

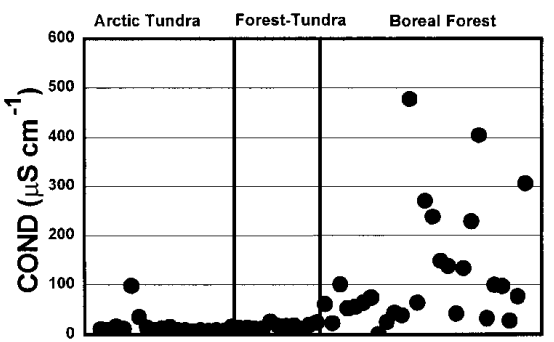

Fig. 2. Trends in selected environmental variables across vegetation zones. Sites are classified into vegetational zones along the $x-$ axis, the value of the measured environmental variable for each lake is given along the y-axis.

It is well known that relatively shallow lakes with small surface areas will be less likely to stratify than deeper larger lakes (Gorham \& Boyce 1989) especially in arctic environments where wind activity can play a major role in lakewater mixing. Thermal stratification may influence (among other things) nutrient distributions within a lake (Gorham \& Boyce 1989). Due to logistical constraints, temperature profiles (surface and bottom) were measured for only 33 (TK-1 to TK-36) of the 56 lakes. Twenty eight of these 33 lakes were weakly to strongly stratified with temperature differences (t.d.) between the surface waters and the bottom waters ranging from 0.2 to $12.4{ }^{\circ} \mathrm{C}$. The majority of lakes in the arctic tundra zone were weakly stratified (mean t.d. $=1.1^{\circ} \mathrm{C}$ ) and 5 of the lakes were isothermal (TK-6, TK-16, TK-19, TK-20, and TK-21). All lakes in the boreal forest and in the forest-tundra showed varying degrees of stratification, six of which were strongly stratified (TK- 25 - t.d. $=5.7^{\circ} \mathrm{C}, \mathrm{TK}-26$ - t.d. $=11.6^{\circ} \mathrm{C}$, TK-28 - t.d. $=9.0^{\circ} \mathrm{C}$, TK-29 - t.d. $=8.7^{\circ} \mathrm{C}$, TK-33 - t.d. $=12.4{ }^{\circ} \mathrm{C}$, and TK-35 - t.d. $=10.9{ }^{\circ} \mathrm{C}$ ). These differences in thermal stratification patterns across the climatic ecozones may be important factors with respect to future climatic warming through changes in the composition of lakewater biota and their habitats.

\subsection{Trends in $p H$ across vegetation zones}

Trends in $\mathrm{pH}$ across ecozones were quite variable, with boreal forest lakes generally recording higher $\mathrm{pH}$ values $(89 \%$ of $\mathrm{pH}$ values higher than 7.0$)$ than arctic tundra lakes $(50 \%$ of $\mathrm{pH}$ values higher than 7.0$)$ (Fig. $2 \mathrm{c}$ ). The $\mathrm{pH}$ range (6.2 to 8.3 with a median of 7.3 ) was somewhat smaller than that recorded in previous studies of this area (Puznicki 1996; Rühland \& Smol 1998) and is likely due to the more uniform nature of the bedrock geology across the sampling transect for this lakeset. It may also indicate that catchment vegetation does not play a major role in affecting lakewater $\mathrm{pH}$ in this lakeset. As expected, $\mathrm{pH}$ was significantly correlated $(\mathrm{p}$ $\leq 0.05)$ to DIC ( $\mathrm{r}=0.55$, $, \mathrm{Mn}(\mathrm{r}=0.40), \mathrm{Mg}(\mathrm{r}=0.49)$, $\mathrm{Ca}(\mathrm{r}=0.52)$, and COND $(\mathrm{r}=0.50)$ (Tab. 3).

\subsection{Trends in major ions, DIC, and conductivity across vegetation zones}

The surface waters of this lakeset were generally dilute with conductivity measurements varying from 2.5 to $478.5 \mu \mathrm{S} \mathrm{cm}^{-1}$, with a median of $10.8 \mu \mathrm{S} \mathrm{cm} \mathrm{c}^{-1}$ in the arctic tundra zone, $18.1 \mu \mathrm{S} \mathrm{cm}^{-1}$ in the forest-tundra zone, and $74.8 \mu \mathrm{S} \mathrm{cm}^{-1}$ in the boreal forest zone (Tab. 4). Despite the dilute nature of these lakes, there was a clear trend in major ions and related variables (such as DIC) across the vegetational gradient (Fig. 2d). Boreal forest lakes were significantly different $(\mathrm{p} \leq 0.05$; Tab. 2 ) and had higher concentrations of $\mathrm{Ca}, \mathrm{Mg}, \mathrm{Na}, \mathrm{K}, \mathrm{Cl}$, $\mathrm{SO}_{4}$, DIC and COND than both arctic tundra and foresttundra lakes. DIC (which partially reflects trends in major ion concentrations) varied from 0.1 to $42.3 \mathrm{mg} \mathrm{l}^{-1}$ with a median of $1.4 \mathrm{mg} \mathrm{l}^{-1}$ in the arctic tundra zone, 1.7 $\mathrm{mg} \mathrm{l}^{-1}$ in the forest-tundra zone, and $8.0 \mathrm{mg} \mathrm{l}^{-1}$ in the boreal forest zone (Tab. 4). This trend of increasing ionic concentrations from arctic tundra to boreal forest lakes has been reported in other treeline studies (Pienitz et al. 1997a, b; Rühland \& Smol 1998; Duff et al. 1998; Fallu \& Pienitz 1999; Gregory-Eaves et al. 2000; Fallu et al. 
2002). This variability in major ion concentrations among zones is likely due to differences in climate-related factors such as hydrology, degree of permafrost, and duration of ice cover (see below).

Patterns in conductivity closely follow those for major ions and DIC (Fig. 2f). For example, Na, K, Ca, $\mathrm{Mg}, \mathrm{Cl}$, as well as DIC, displayed high and significant ( $\mathrm{p} \leq 0.01$ ) correlations with COND (Tab. 3). Sites with higher COND measurements (100.2 to $478.5 \mu \mathrm{S} \mathrm{cm}^{-1}$ ) had consistently higher DIC (8.0 to $\left.42.3 \mathrm{mg} \mathrm{l}^{-1}\right), \mathrm{Ca}(7.7$ to $\left.34.7 \mathrm{mg} \mathrm{l}^{-1}\right), \mathrm{Mg}\left(2.7\right.$ to $\left.32.4 \mathrm{mg} \mathrm{l}^{-1}\right), \mathrm{K}$ (1.6 to 16.8 $\left.\mathrm{mg} \mathrm{l}^{-1}\right), \mathrm{Cl}\left(1.1\right.$ to $\left.33.4 \mathrm{mg} \mathrm{l}^{-1}\right), \mathrm{Na}\left(1.8\right.$ to $\left.39.8 \mathrm{mg} \mathrm{l}^{-1}\right)$, and $\mathrm{SO}_{4}\left(2.6\right.$ to $\left.13.3 \mathrm{mg} \mathrm{l}^{-1}\right)$ values, all of which were located in the boreal forest zone. Lakes with particularly high COND values $\left(>150 \mu \mathrm{S} \mathrm{cm} \mathrm{cm}^{-1}\right.$ ) were located in proximity to each other and to the city of Yellowknife (TK-37, TK-39, TK-40. TK-45, TK-46, TK-59), likely as a result of their location closer to human activity. The correlation between COND and DIC is particularly strong $(r=0.89, p \leq 0.01)$. Similar to other arctic studies (Pienitz et al. 1997a, b; Rühland \& Smol 1998; Gregory-Eaves et al. 2000; Lim et al. 2001), the general trend in relative concentrations of cations was $\mathrm{Ca}>\mathrm{Mg}>\mathrm{Na}>\mathrm{K}$ with mean values of $6.8 \mathrm{mg} \mathrm{l}^{-1}, 3.8 \mathrm{mg}$ $\mathrm{l}^{-1}, 2.6 \mathrm{mg} \mathrm{l}^{-1}$, and $1.7 \mathrm{mg} \mathrm{l}^{-1}$, respectively (Tab. 4). The relative concentrations in major anions followed similar trends to Lim et al. (2001) with DIC (most of which is bicarbonate $\left(\mathrm{HCO}_{3}\right)$ given the circumneutral $\mathrm{pH}$ range of this dataset) $>\mathrm{SO}_{4}>\mathrm{Cl}$ with mean values of $6.5 \mathrm{mg}$ $\mathrm{l}^{-1}, 3.4 \mathrm{mg} \mathrm{l}^{-1}$, and $3.1 \mathrm{mg} \mathrm{l}^{-1}$, respectively (Tab. 4).

The decrease in ionic concentrations from south to north may be indirectly related to climatic factors. As bedrock geology across this vegetational gradient is relatively uniform, other factors are likely responsible for differences in major ion variations across the climatic gradient. Lakes situated in the continuous permafrost zone (arctic tundra sites) will have much more restricted groundwater inflow resulting in a lower net yield of base cations entering the lakes than those situated on discontinuous permafrost to the south. Higher values in DIC, and related variables in boreal forest lakes may also be related to higher precipitation and runoff than in arctic tundra sites (Schindler et al. 1996; Henriksen et al. 1998). Pienitz et al. (1997a, b) have found similar trends in their lakesets spanning treeline in both the Yukon and the Northwest Territories. They attributed the higher values in the forested catchments to increased weathering and ion supply from the catchment as a result of higher precipitation. Thicker and more highly developed soils in the boreal forest also contribute to the higher ionic concentrations in this vegetation zone. In addition, in-lake alkalinity generation, such as from increased biological productivity and sulfate reduction processes, are more pronounced in warmer climates (Webster et al. 1990; Psenner \& Schmidt 1992; Schindler et al. 1996) such as is found in the boreal forest zone.

\subsection{Trends in DOC across vegetation zones}

A wide range in DOC concentrations was measured in this lakeset with a minimum of $1.3 \mathrm{mg} \mathrm{l}^{-1}$ to a maximum of $332.0 \mathrm{mg} \mathrm{l}^{-1}$, with a median of $13.4 \mathrm{mg} \mathrm{l}^{-1}$ (Tab. 4). This range was about an order of magnitude greater than those reported by Pienitz et al. (1997a, b), Moser et al. (1998), Duff et al. (1998), and Fallu \& Pienitz (1999), but similar to Alaskan values reported by Gregory-Eaves et al. (2000).

Lower DOC values were recorded in arctic tundra lakes (median $=2.3 \mathrm{mg} \mathrm{l}^{-1}$ ), than in lakes within the boreal forest zone $\left(\right.$ median $=26.2 \mathrm{mg} \mathrm{l}^{-1}$ ) (Fig. 2e). This trend of increasing DOC concentrations from forest to tundra was expected as waters draining the catchments of coniferous leaf litter bring high amounts of allochthonous DOC into lakewaters (Wetzel 1983; Cronan \& Aiken 1985; Forsberg 1992; Schindler et al. 1992; Hongve 1999). Additionally, wetlands, commonly found in the study region provide another important allochthonous source of DOC (Henriksen et al. 1998). Water colour in many of the boreal forest and foresttundra lakes was commonly tea-coloured to dark chocolate-coloured, a strong indication of high levels of catchment-derived DOC comprised of coloured humic substances (Meili 1992). For example, boreal forest lakes that were reported to be very darkly coloured (TK35, TK-28, TK-33, TK-36, TK-37, TK-39, TK-41, TK46) had corresponding high levels of DOC (51.6 to $\left.332.0 \mathrm{mg} \mathrm{l}^{-1}\right)$. This large gradient in lakewater DOC between treeless and forested catchments suggest that potentially this variable may track past changes in coniferous forests in this region.

\subsection{Trends in nutrients and chlorophyll-a across the vegetational gradient}

Nutrients were expected to track changes in the different vegetation zones as one primary source of nutrients to lakes is from terrestrial sources (Guildford \& Hecky 2000). Higher allochthonous sources of nutrients entering into forest lakes probably result from higher runoff, higher precipitation, and denser vegetation. In this forested zone, increased breakdown of terrestrial litter, a longer growing season (i.e. shorter duration of ice cover) in combination with warmer temperatures would elevate primary productivity and intensify nutrient circulation. In the arctic tundra, shorter growing seasons, cooler conditions, restricted hydrological processes, continuous permafrost, and diminished catchment-derived nutrients would likely reflect lower primary productivity.

The majority of our study lakes were oligotrophic but did display a moderate range in trophic status (North American standards) from ultra-oligotrophic (TPUF $<5.0 \mu \mathrm{g} \mathrm{l}^{-1}, 11$ lakes) to eutrophic (TPUF $>30 \mu \mathrm{g} \mathrm{l}^{-1}, 3$ lakes). In general, boreal forest lakes had higher TPUF concentrations (median $=10.8 \mu \mathrm{g} \mathrm{l}^{-1}$ and a range of 3.7 to $48.6 \mu \mathrm{g} \mathrm{l}^{-1}$ ) than arctic tundra lakes (median $=6.1 \mu \mathrm{g}^{-1}$ 
a)

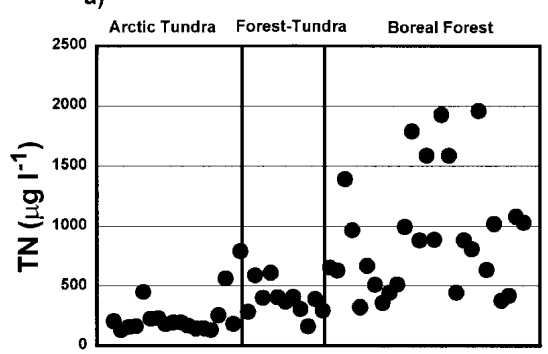

d)

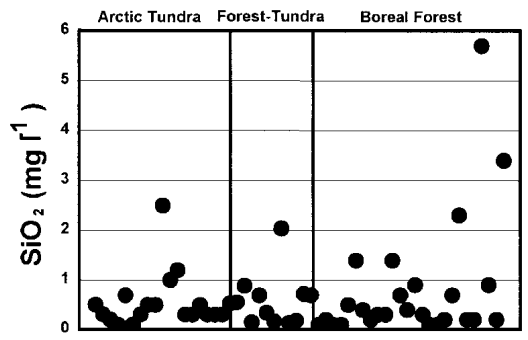

b)

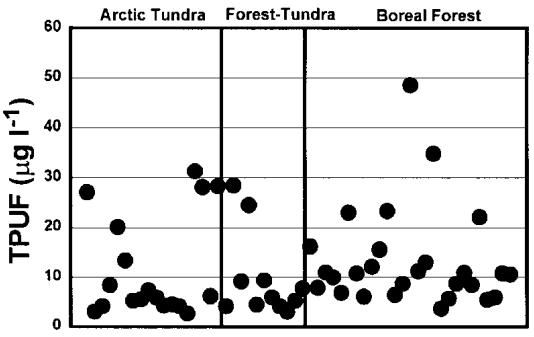

e)

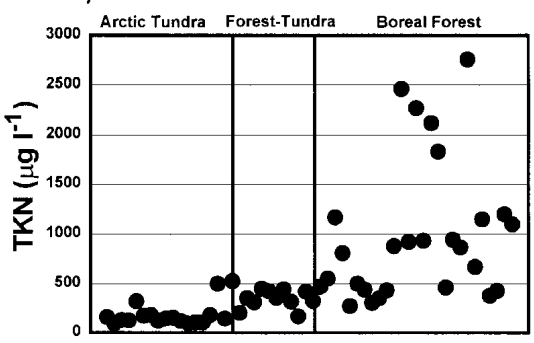

c)

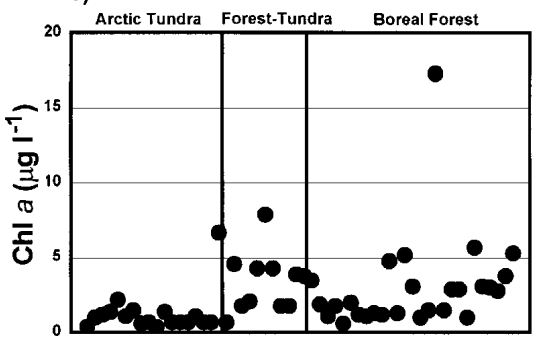

f)

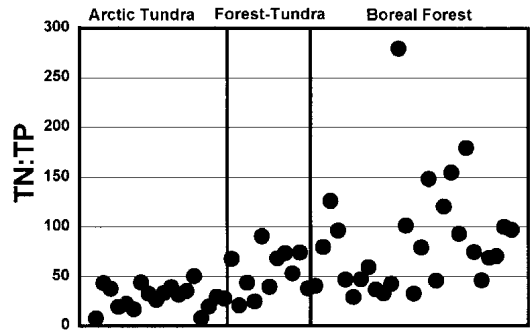

Fig. 3. Trends in nutrients and CHLA across vegetation zones. Sites are classified into zones along the x-axis, the value of the measured environmental value for each lake is given along the y-axis.
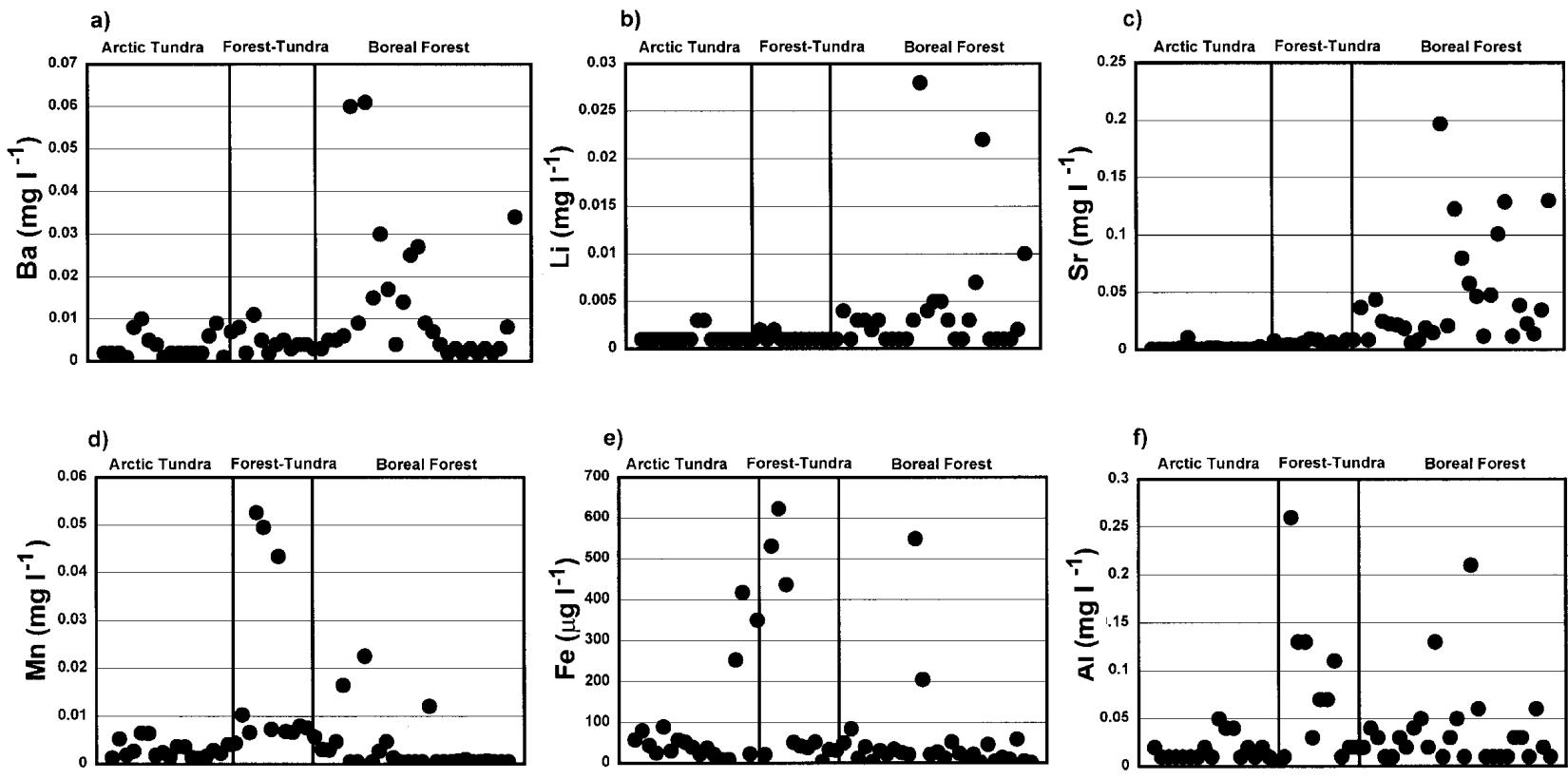

e)

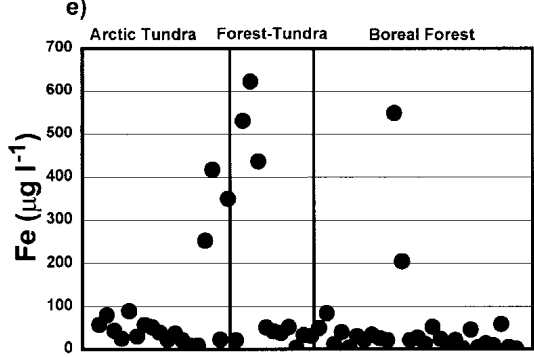

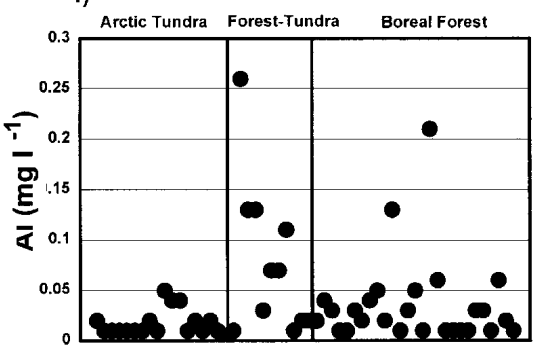

Fig. 4. Trends in metal elements across vegetation zones. Sites are classified into zones along the $x$-axis, the value of the measured environmental variable for each lake is given along the y-axis.

and a range of 2.7 to $31.3 \mu \mathrm{g} \mathrm{l^{-1 }}$ ) and forest-tundra lakes (median $=6.0 \mu \mathrm{g} \mathrm{l}^{-1}$ and a range of 3.1 to $28.5 \mu \mathrm{g} \mathrm{l}^{-1}$ ) (Tab. 4, Fig. 3b). According to this classification system, three lakes were classified as eutrophic, two of which were located in the boreal forest (TK-39: $48.6 \mu \mathrm{g}$ $1^{-1} \&$ TK-42: $34.8 \mu \mathrm{g} \mathrm{l}^{-1}$ ) and one in the arctic tundra (TK-18: $31.3 \mu \mathrm{g} \mathrm{l}^{-1}$ ). Both boreal forest lakes were very shallow (about $1.0 \mathrm{~m}$ in depth) and were relatively close to the city of Yellowknife (approximately $25 \mathrm{~km}$ ). Reasons for the higher phosphorus values are not clear, but may be related to the possibility that these lakes receive water from nearby Prelude Lake to the north, which has a small lodge (accommodates 15 people) and a small community campsite (28 sites) (Wedel et al. 1990) that may be a potential source of nutrients. The CHLA value for TK-42 (17.3 $\left.\mu \mathrm{g} \mathrm{l}^{-1}\right)$ was the highest recorded for the entire lakeset, but for TK-39 CHLA levels were moderately low $\left(3.1 \mu \mathrm{g} \mathrm{l}^{-1}\right)$. The higher value for TPUF in the arctic tundra lake (TK-18) as well as in a lake a few meters away (TK-19) may be related to the relatively 
high iron concentrations (253.0 and $418.0 \mu \mathrm{g} \mathrm{l}^{-1}$ ). Both lakes were dark red in colour. Iron is known to form complexes with phosphorus, leaving the measured phosphorus unavailable for biological uptake (Jones et al. 1988). This appears to be the case for these lakes, as low algal biomass, as inferred from CHLA, were also recorded (TK-18: CHLA $=1.1 \mu \mathrm{g} \mathrm{l}^{-1}$; TK-19: CHLA = $0.7 \mu \mathrm{g} \mathrm{l}^{-1}$ ). Therefore, even though the phosphorus levels are high, the lakes are still not very productive.

Nutrient concentrations and CHLA followed similar spatial trends, as expected, with higher concentrations in $\mathrm{SiO}_{2}, \mathrm{CHLA}, \mathrm{TKN}, \mathrm{TN}$, and TPUF measured in boreal forest lakes (medians of $0.3 \mathrm{mg} \mathrm{l}^{-1}, 2.0 \mu \mathrm{g} \mathrm{l}^{-1}, 869.0 \mu \mathrm{g}$ $\mathrm{l}^{-1}, 883.0 \mu \mathrm{g} \mathrm{l}^{-1}$, and $10.8 \mu \mathrm{g} \mathrm{l}^{-1}$, respectively) than in arctic tundra lakes (medians of $0.3 \mathrm{mg} \mathrm{l}^{-1}, 0.9 \mu \mathrm{g} \mathrm{l}^{-1}$, $145.5 \mu \mathrm{g} \mathrm{l}^{-1}, 192.0 \mu \mathrm{g} \mathrm{l}^{-1}$, and $6.1 \mu \mathrm{g} \mathrm{l}^{-1}$, respectively) (Tab. 4, Fig. 3). Our values were similar to those reported by Lim et al. (2001) and by Gregory-Eaves et al. (2000), but had substantially higher concentrations of TKN, TN, and TPUF than other Canadian treeline studies (Pienitz et al. 1997a; Fallu \& Pienitz 1999).

CHLA was not significantly correlated to other measured variables with the exception of nitrogen (TKN and TN) (Tab. 3). TPUF and CHLA were only weakly correlated $(\mathrm{r}=0.31)$ suggesting that productivity in some of these lakes may be limited by other factors, such as nitrogen.

To determine whether the POC of our lakes was primarily derived from allochthonous versus autochthonous sources, a ratio of POC (particulate organic carbon) to CHLA (representing algal biomass) was calculated for all lakes where POC measurements were available $(\mathrm{n}=33)$. These ratios were also explored by Lim et al. (2001) and by Hamilton et al. (2001) for Canadian high arctic sites. In our reduced lakeset of 33 sites, the POC:CHLA ratios ranged from 170:1 to 2973:1, with a mean of 616:1 (Tab. 4). Eppley et al. (1977) suggested that ratios greater than 100 are indicative of higher loading of detrital POC coming into the lake from external sources (i.e. not from algal sources within the lake). To further explore the relationship between these two indicators of production, linear regressions between CHLA and POC for boreal forest lakes and for arctic tundra lakes (only 4 sites in the forest-tundra had POC data available and was therefore not included here) were generated (Fig. 5a-c). Weak relationships were found between POC and CHLA for lakes in the arctic tundra ecozone $\left(r^{2}=0.02, n=16\right.$ after the removal of two outliers), as well as for lakes in the boreal forest $\left(\mathrm{r}^{2}=0.20\right.$, $\mathrm{n}=10$ after the removal of one outlier). The higher ratio of POC:CHLA in our boreal forest lakes $($ mean $=824.3)$ than our arctic tundra lakes $($ mean $=456.0)$ together with the poor relationships observed suggest that lakes in our forested catchments have higher loadings of detrital matter than do arctic tundra lakes. This would be expected, as increased catchment vegetation and richer soils can contribute significant amounts of detrital POC in comparison to the more sparsely vegetated catchments with poorly developed soils of arctic tundra sites.

The relationship between POC and CHLA in the arctic tundra zone can be compared to the ratios calculated by other researchers in the Canadian Arctic and Subarctic (Pienitz et al. 1997a, b; Lim et al. 2001; Hamilton et al. 2001) and in Alaska (Gregory-Eaves et al. 2000). As in this dataset, the majority of lakes in these studies $(98 \%, \mathrm{n}=91)$ had POC:CHLA ratios well above 100 , suggesting that even these tundra lakes received much of their detrital loading from allochthonous sources. Much like this study, linear regressions of the data from these other studies did not reveal strong relationships between these two variables for lakes in the arctic tundra, with the exception of lakes in Alaska $\left(\mathrm{r}^{2}=\right.$ $0.87, \mathrm{n}=9$ ).

To assess whether our lakes were primarily limited by phosphorus or by nitrogen, TN:TP ratios were calculated. TN:TP ratios for this lakeset ranged from 7.7 to 279.7 with a mean of 61.8 (Tab. 4). Nitrogen limitation may be common in lakes with TN:TP ratios less than 14 (especially if TP was high) (Downing \& McCauley 1992) and tend to be phosphorus-limited in lakes with ratios greater than 17 (Sakomoto 1966). Using these criteria, only two lakes from this study (TK-1 and TK18; both in the arctic tundra zone) were classified as possibly nitrogen-limited and both had TPUF concentrations well above the mean $\left(27.1 \mu \mathrm{g}^{-1}\right.$ and $31.3 \mu \mathrm{g} \mathrm{l}^{-1}$, respectively). All other lakes were classified as phosphorus-limited. The findings reported here agree with Pienitz et al. (1997a), Gregory-Eaves et al. (2000), and Hamilton et al. (2001). There is a clear spatial trend in this ratio with an increase in TN:TP from the arctic tundra to the boreal forest (Fig. 3f). This is not surprising, as higher TN:TP values are to be expected in lakes receiving runoff from undisturbed forested watersheds than lakes that depend more on inputs from precipitation such as in the arctic tundra (Downing \& McCauley 1992). However, these calculations should be viewed with caution, as this dataset does not provide extensive year-round sampling, but only one snapshot sample for each lake collected in August.

\subsection{Trends in trace metals across vegetation zones}

Concentrations of aluminum, barium, iron, lithium, manganese and strontium were all within the range considered common for natural surface waters in Canada $\left(<1000 \mu \mathrm{g} \mathrm{l}^{-1},<1000 \mu \mathrm{g}^{-1},<500 \mu \mathrm{g} \mathrm{l}^{-1},<200\right.$ $\mu \mathrm{g}^{-1},<1000 \mu \mathrm{g} \mathrm{l}^{-1}$, respectively; McNeely et al. 1979). All of these metals (with the exception of Fe) had significantly higher $(\mathrm{p}<0.05)$ concentrations in boreal forest lakes than in forest-tundra or arctic tundra lakes (Tab. 2), and closely followed the trends in major ions and related variables (Fig. 4).

Concentrations of $\mathrm{Ba}, \mathrm{Sr}$, and $\mathrm{Li}$ were significantly higher $(\mathrm{p}<0.05)$ in the boreal forest (medians of 0.008 $\mathrm{mg} \mathrm{l}^{-1}, 0.025 \mathrm{mg} \mathrm{l}^{-1}$, and $0.003 \mathrm{mg} \mathrm{l}^{-1}$, respectively) and 

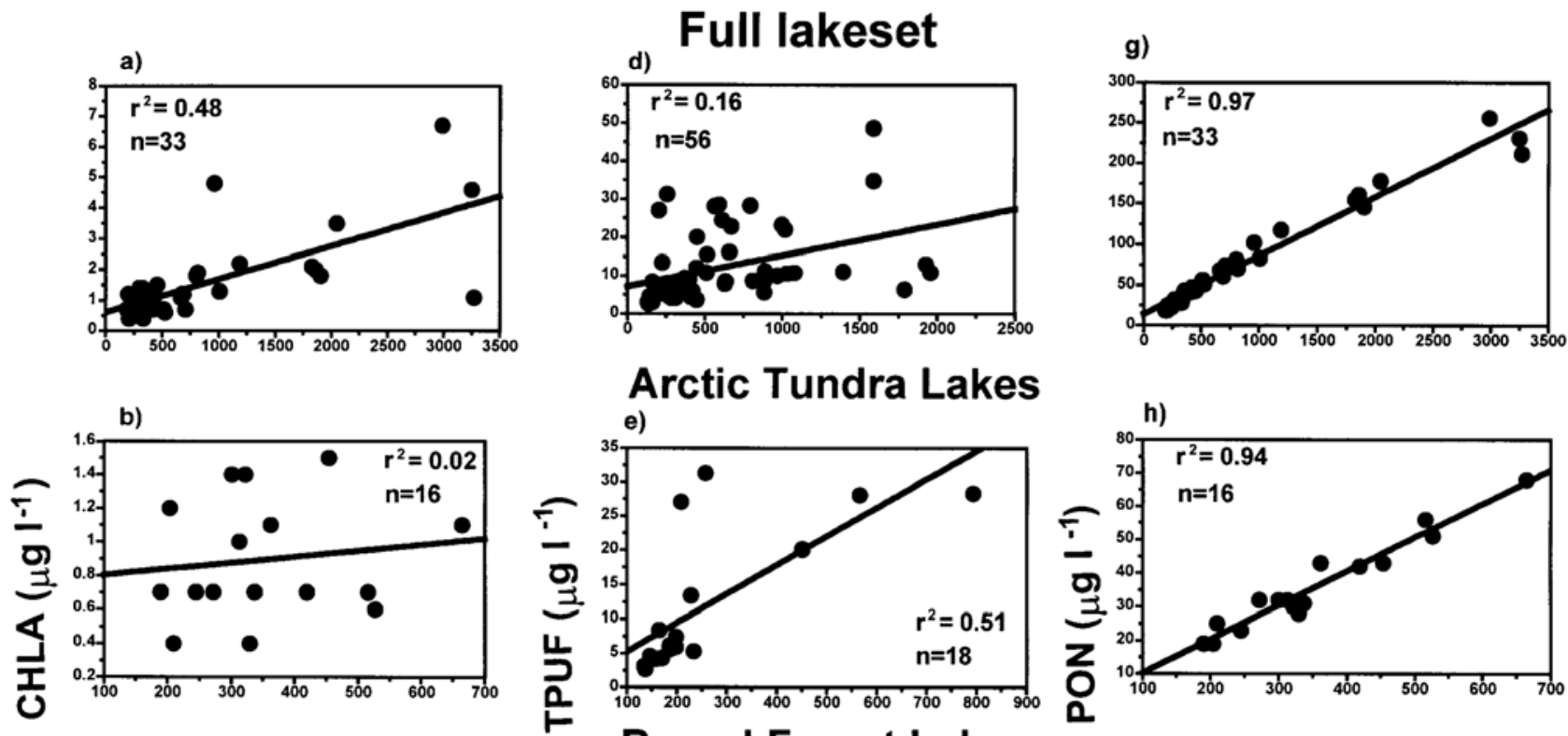

Arctic Tundra Lakes
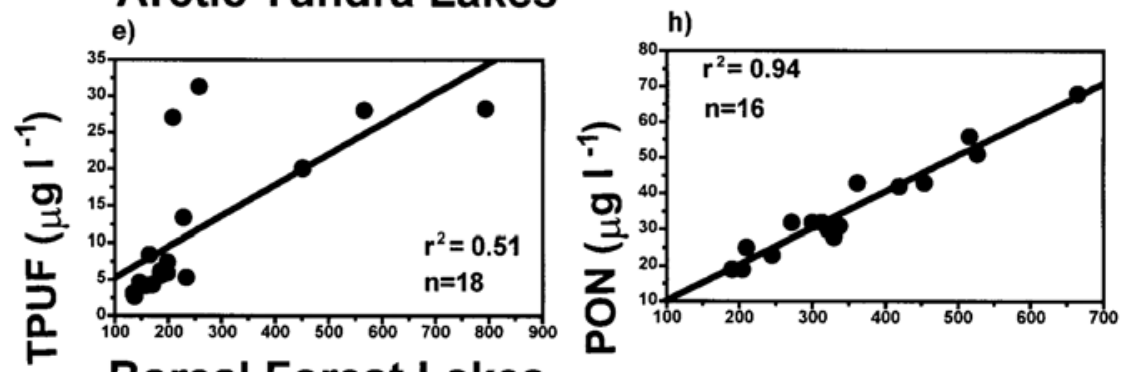

c)
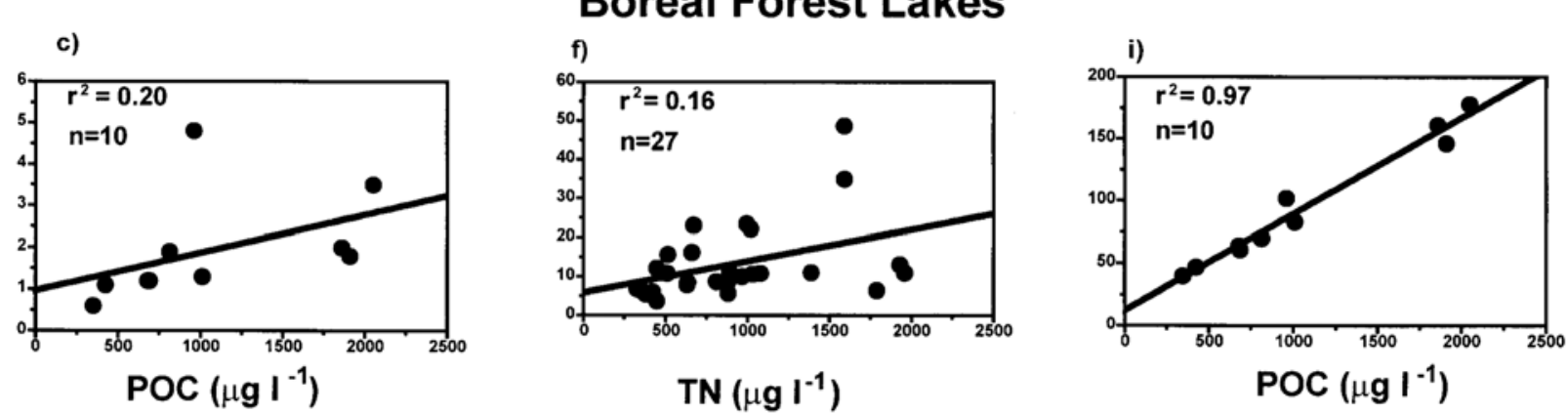

Fig. 5. Comparison of selected nutrient relationships between arctic tundra and boreal forest lakes.

lower in the arctic tundra (medians of $0.002 \mathrm{mg} \mathrm{l}^{-1}$, $0.001 \mathrm{mg} \mathrm{l}^{-1}$, and $0.001 \mathrm{mg} \mathrm{l}^{-1}$, respectively) (Tab. 2, Tab. 4). All three of these metallic elements had significant and positive correlations to major ions and DIC (Tab. 3). The values for Li were consistent with values reported by Puznicki (1996), Moser et al. (1998), Gregory-Eaves et al. (2000), and Lim et al. (2001). Not surprisingly, Li was most highly correlated to $\mathrm{Na}$ and $\mathrm{K}(\mathrm{r}=0.81, \mathrm{p} \leq 0.01$; Tab. 3$)$, as all three are univalent alkali metals. However, concentrations of $\mathrm{Ba}$ and $\mathrm{Sr}$ were substantially lower in this lakeset than values reported previously. This may be due to the scarcity of calcium-carbonate and magnesium-rich deposits and the more granitic nature of the surrounding bedrock, as higher values of these metals are expected in lakes whose catchments drain rocks richer in calcium carbonate. Puznicki (1996) reported notably higher concentrations of $\mathrm{Ba}$ and $\mathrm{Sr}$ (maximum of $0.083 \mathrm{mg} \mathrm{l}^{-1}$ and $0.177 \mathrm{mg} \mathrm{l}^{-1}$, respectively) in lakes around Great Bear Lake, where calcium carbonate-rich deposits are more predominant. Unlike our study, calcium carbonate-rich deposits were also reported in many of the sites studied by the other researchers mentioned above.
Although iron concentrations were within the range commonly found in Canadian surface waters (McNeely et al. 1979), several lakes (TK-18, TK-19, TK-21, TK-30, TK-31, TK-32, TK-36, TK-37) were dark orangy/ reddish-brown in colour and had iron values $\left(205.0-623.0 \mu \mathrm{g} \mathrm{l}^{-1}\right)$ well above the lakeset mean $\left(86.8 \mu \mathrm{g}^{-1}\right)$. As DOC concentrations were relatively low for most of these lakes (mean $29.9 \mathrm{mg}$ $1^{-1}$ ), the coloured nature of these lakes may be a consequence of iron-rich deposits within their catchments. $\mathrm{Fe}$ and $\mathrm{Mn}$ are often linked to high concentrations of humic materials and therefore, lakes with high concentrations of DOC often record higher values of $\mathrm{Fe}$ and $\mathrm{Mn}$ (e.g. Moser et al. 1998; Fallu \& Pienitz 1999). Unlike these other arctic studies, no such relationship was observed in this lakeset $(\mathrm{r}=$ $0.02, \mathrm{r}=0.11$, respectively; Tab. 3 ). The only measured variable that was significantly correlated to Mn was $\mathrm{pH}(\mathrm{r}=0.49, \mathrm{p} \leq 0.05$; Tab. 3$)$. This is likely due to $\mathrm{Mn}$ being brought into solution through weathering processes such as acid leaching (McNeely et al. 1979). 


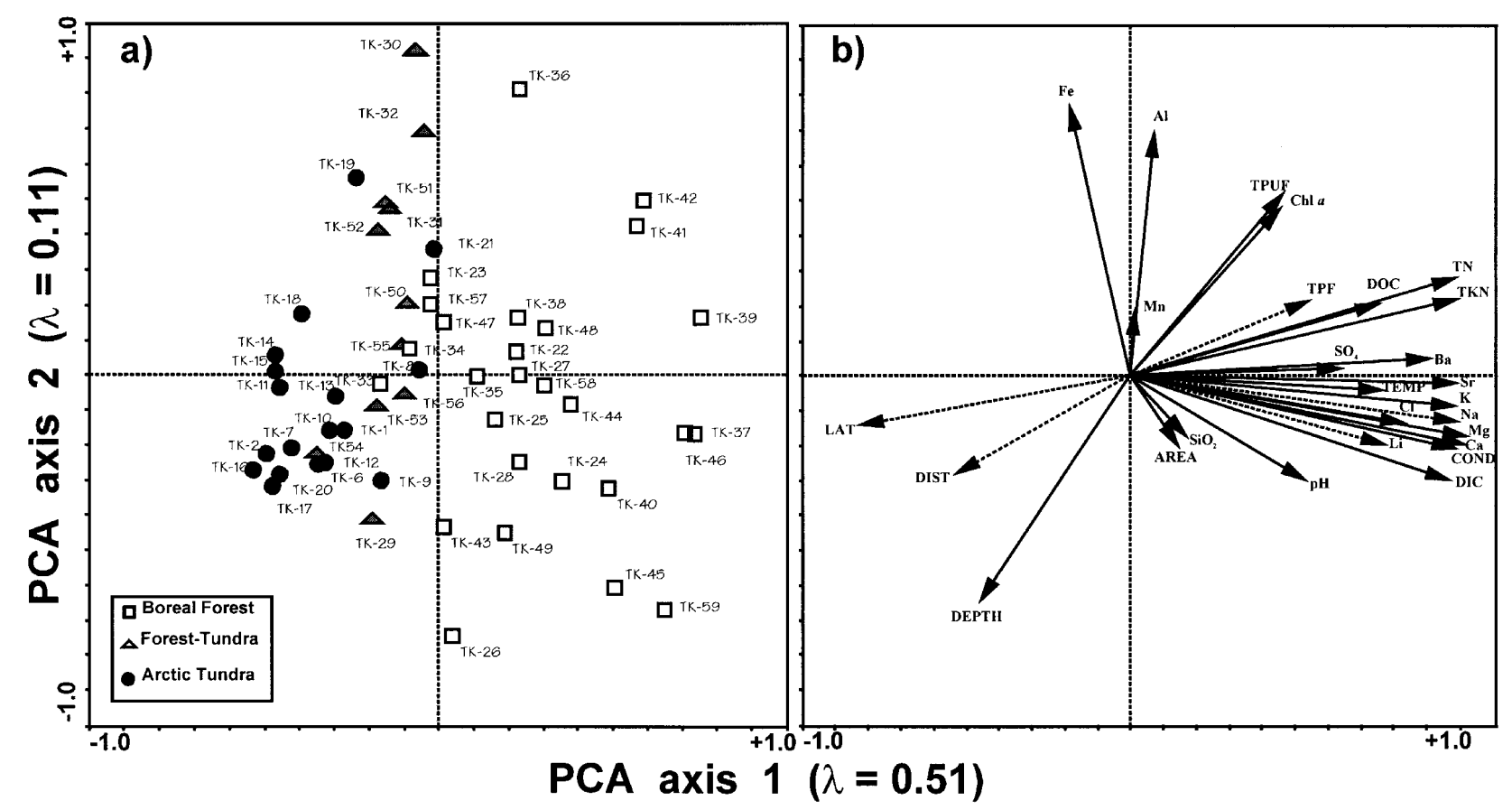

Fig. 6. Principal Components Analysis (PCA) of the 56 study sites and environmental variables. Dashed lines represent environmental variables that were run passively in the ordination.

\subsection{Ordination (PCA)}

The distribution of variables and sites in the ordination closely tracked the patterns across vegetational zones discussed earlier. PCA revealed two main directions of variation, with axis 1 explaining $51 \%$ of the total variance and axis 2 explaining an additional $11 \%$ (Fig. 6). An examination of the inter-set correlations of the 20 active environmental variables, together with the position and length of the arrows, revealed that the strongest direction of variation (axis 1) was primarily a gradient of major ions and nitrogen consisting of $\mathrm{Ca}$, $\mathrm{Mg}, \mathrm{K}, \mathrm{Cl}$, SO4, COND, pH, DIC, DOC, nitrogen TKN, TN, Ba, Sr, and TEMP. All of these variables had high positive correlations with PCA axis 1 and to each other (Tab. 3), and plotted towards the right of the ordination. The second gradient (axis 2) was characterized by strong inter-set correlations to $\mathrm{Al}, \mathrm{Fe}, \mathrm{TPUF}$, and DEPTH. Al and Fe were positively correlated to PCA axis 2 and to each other, and negatively correlated to DEPTH (Tab. 3, Fig. 6).

The 56 sites were clearly separated into the three ecozones along the first PCA gradient, with boreal forest lakes plotting almost exclusively on the right hand side of the ordination, indicating that these sites were characterized by higher concentrations of major and minor ions (and related variables) and nitrogen (Fig. 6a). Arctic tundra sites, on the other hand, plotted exclusively on the left hand side of the ordination, and were associated with more dilute lakewater chemistry. Boreal forest sites showed greater dispersal in the PCA ordina- tion space than did the arctic tundra sites, reflecting greater variability in lakewater chemistry within this ecozone. The second direction of variation was less obviously related to the vegetational gradient. Here, deeper lakes plotted towards the lower half of the ordination, and lakes with higher concentrations in $\mathrm{Fe}$ and Al plotted towards the upper half of the plot. As there were no obvious spatial trends for DEPTH, Fe or Al, (Fig. 2a, Fig. 4ef), sites that plotted near these environmental arrows in the PCA did not show any clear association to vegetation zones (Fig. 6a, b).

\subsection{Some implications of treeline spatial trends in terms of predicted climatic warming}

The spatial gradient of climate-related factors (e.g. temperature, vegetation, precipitation, degree of permafrost) across treeline was often closely reflected in the lakewater chemistry of the 56 study lakes. Therefore, these limnological trends may provide important information on possible future changes with additional climatic warming. For example, warmer climates will shorten the duration of ice cover on lakes. This could result in some currently polymictic or isothermal lakes to thermally stratify, and dimictic lakes to have longer and more stable periods of thermal stratification (De Stasio et al. 1996). The length of the growing season will also increase with diminished ice and snow cover, extending the photosynthetically active period and increasing algal production rates (Smol 1983, 1988; Douglas \& Smol 1999). A warmer and drier climate will affect the hydrological regime, as increases in evapora- 
tion will not likely be compensated for by increases in precipitation (Schindler et al. 1997). Changes in the extent of permafrost (deeper active layer and continuous permafrost becoming discontinuous in northern regions) will also have a profound effect on the hydrological system through increased groundwater flow and runoff from the catchment to the lake.

The above physical changes brought about by climatic warming will have important implications on lakewater chemistry. Increased runoff and groundwater flow should increase the input of nutrients to lakes, resulting in higher production. The net yield of base cations entering the lakes should increase as a result greater groundwater inputs, which should also increase pH (Webster et al. 1990; Schindler et al. 1996). Increases in $\mathrm{pH}$ are also expected during warmer temperatures through enhanced in-lake alkalinity generation processes such as increased biological productivity and stronger sulfate reduction processes (Psenner \& Schmidt 1992; Schindler et al. 1996). These physical and chemical changes, will undoubtedly have important impacts on aquatic habitats and species composition in freshwater ecosystems.

\section{CONCLUSIONS}

The chemical characteristics of the 56 study lakes appear to be strongly influenced by climatic and climate-related factors (e.g. degree of permafrost, vegetation, weathering, duration of snow and ice cover). Our results show that: 1) distinct limnological differences exist among treeline ecozones; 2) of all the measured limnological variables, ionic and nutrient concentrations displayed the greatest changes from the subarctic boreal forest to the arctic tundra; and 3) arctic tundra lakes were more chemically dilute than subarctic forested lakes.

Comparisons with other circumpolar treeline regions indicate that some global patterns are emerging, suggesting that climatic warming will impact the chemistry of freshwater treeline lakes. However, because lakewater chemistry can also be related to factors other than climate (e.g. local geology, vegetation, human disturbances and physiographic relief) and due to the vastness of this ecotone on a global scale, more studies of this kind are needed to refine our understanding of baseline limnological conditions at circumpolar treeline. Future research should include monitoring of treeline lakes to examine the changing patterns of ice cover duration, thermal stratification, and the associated changes in lakewater limnology. From a paleoclimatic perspective, studies of this kind, coupled with paleolimnological analyses, have the potential to be used to track past shifts in the arctic treeline and other climate-related characteristics. These data will provide important information needed to better understand these changing ecosystems under a climatic warming scenario.

\section{ACKNOWLEDGMENTS}

This research has been supported by an NSERC grant to J.P. Smol, a Northern Studies Training Program (NSTP) grant, as well as an NSERC special collaborative grant to Paleoecological Analysis of Circumpolar Treeline (PACT). Logistic support from the Polar Continental Shelf Project (PCSP) is also gratefully acknowledged (PCSP contribution \# 02502). We are also grateful for a Queen's University Dean's Travel award that helped make it possible to collect additional samples (TK-37 to TK-59). We would also like to thank T. Karst, T. Laing, and B. Wolfe for assistance in sampling these lakes. We would like to thank the Department of Indian Affairs and Northern Development in Yellowknife (TAIGA Laboratories) for allowing us to store our samples at their facilities while in the field, and for coming to our aid when we had equipment problems. We thank John Glew for drafting our map, Scott Lamoureux, Sherri Cooper, Brian Cumming, and three anonymous reviewers for improving the clarity and quality of this paper.

\section{REFERENCES}

Blom, T., A. Korhola, J. Weckström, T. Laing, J. Snyder, G. MacDonald \& J.P. Smol. 1999. Physical and chemical characterisation of small subarctic headwater lakes in Finnish Lapland and the Kola Peninsula. Verh. int. Ver. Limnol., 27: 1-5.

Bonan, G.B., F.S. Chapin III \& S.L. Thompson. 1995. Boreal forest and tundra ecosystems as components of the climate system. Climatic Change, 29: 145-167.

Canada Department of Energy, Mines and Resources. 1991. National Atlas of Canada. 5th ed., Department of Energy, Mines and Resources, Ottawa, Canada.

Canada. Natural Resources Canada, Canadian Forest Service, and Geomatics Canada. 1996. National Atlas of Canada: The Boreal Forest.

Clayton, J.S., W.A. Ehrlich, D.B. Cann, J.H. Day \& I.B. Marshall. 1977. Soils of Canada, Volume II. Ottawa: Soil Inventory Research Branch, Canada, Department of Agriculture: $239 \mathrm{pp}$.

Cronan, C.S. \& G.R. Aiken. 1985. Chemistry and transport of soluble humic substances in forested watersheds of the Adirondack Park, New York. Geochimica et Cosmochimica Acta, 49: 1607-1705.

Cumming, B.F., S.E. Wilson, R.I. Hall \& J.P. Smol. 1995. Diatoms from British Columbia (Canada) Lakes and their Relationship to Salinity, Nutrients, and Other Limnological Variables. Bibliotheca Diatomologica: 207 pp.

DeStasio, B.T., J.M. Hill, N.P. Kleinhans, N.P. Nibbelink \& J.J. Magnuson. 1996. Potential effects of global climate change on small north temperate lakes: physics, fish and plankton. Limnol. Oceanogr., 41: 1136-1149.

Douglas, M.S.V. \& J.P. Smol. 1999. Freshwater diatoms as indicators of environmental change in the High Arctic. In: Stoermer, E. \& J.P. Smol (Eds), The Diatoms: Applications for the Environmental and Earth Sciences. Cambridge University Press, Cambridge, U.K.: 227-244.

Downing, J.A. \& E. McCauley. 1992. The nitrogen : phosphorus relationship in lakes. Limnol. Oceanogr., 37: 936-945.

Duff, K.E., T.E. Laing, J.P. Smol \& D.R.S. Lean. 1998. Limnological characteristics of lakes located across arctic treeline in Northern Russia. Hydrobiologia, 391: 205-222. 
Environment Canada. 1990. The Climates of Canada. Ottawa: Canadian Government Publishing Centre: 176 pp.

Environment Canada. 1994a. Manual of Analytical Methods: Major ions and nutrients, volume 1. National Laboratory for Environmental Testing, Canadian Centre for Inland Waters, Burlington, Ontario: 651pp.

Environment Canada. 1994b. Manual of Analytical Methods: Trace Metals, volume 2. National Laboratory for Environmental Testing, Canadian Centre for Inland Waters, Burlington, Ontario: 446pp.

Eppley, R.W., W.G. Harrison, S.W. Chisholm \& E. Stewart. 1977. Particulate organic matter in surface waters off California, and its relation to photosynthesis. J. Marine Res., 35: 671-696.

Fallu, M.-A. \& R. Pienitz. 1999. Diatomeés lacustres de Jamésie-Hudsonie (Québec) et modèle de reconstitution des concentrations de carbone organique dissous. Ecoscience, 6: 603-620.

Fallu, M.A., N. Allaire \& R. Pienitz. 2002. Distribution of freshwater diatoms in 64 Labrador (Canada) lakes: species-environment relationships along latitudinal gradients and reconstruction models for water colour and alkalinity. Can. J. Fish. Aquat. Sci., 59: 329-349.

Foley, J.A., J.E. Kutzbach, M.T. Coe \& S. Levis. 1994. Feedbacks between climate and boreal forests during the Holocene epoch. Nature, 371: 52-54.

Forsberg, C. 1992. Will an increased greenhouse impact in Fennoscandia give rise to more humic and coloured lakes? Hydrobiologia, 229: 51-58.

Gorham, E. \& F.M. Boyce. 1989. Influence of lake surface area and depth upon thermal stratification and the depth of the summer thermocline. J. Great Lakes Res., 15: 233-245.

Gregory-Eaves, I., J.P. Smol, B.P. Finney, D.R.S. Lean \& M.E. Edwards. 2000. Characteristics and variation in lakes along a north-south transect in Alaska. Arch. Hydrobiol., 147: 193-223

Guilford, S.J. \& R.E. Hecky. 2000. Total nitrogen, total phosphorus, and nutrient limitation in lakes and oceans: Is there a common relationship? Limnol. Oceanogr., 45: 1213-1223.

Hamilton, P.B., K. Gajewski, D. Atkinson \& D.R.S. Lean. 2001. Physical and chemical limnology of 204 lakes from the Canadian Arctic Archipelago. Hydrobiologia, 457: 133-148.

Healey, M.C. \& W.L. Woodall. 1973. Limnological surveys of seven lakes near Yellowknife, Northwest Territories. Technical Report No. 407, Fisheries Research Board of Canada.

Henrkisen, A., B.L. Skjelvåle, J. Mannio, A. Wilander, R. Harriman, C. Curtis, J.P. Jensen, E. Fjeld \& T. Moiseenko. 1998. Northern European Lake Survey, 1998. Ambio, 27(2): 80-91.

Hongve, D. 1999. Production of dissolved organic carbon in forested catchments. J. Hydrol., 224: 91-99.

Jones, R.I., K. Salonen \& H. De Haan. 1988. Phosphorus transformations in the epilimnion of humic lakes: abiotic interactions between dissolved humic materials and phosphate. Freshwat. Biol., 19: 357-369.

Juggins, S. \& C.J.F. ter Braak. 1993. CALIBRATE - unpublished computer program, Environmental Change Research Centre, University College, London.

Lim, D.S.S., M.S.V. Douglas, J.P. Smol. \& D.R.S. Lean. 2001. Physical and chemical limnological characteristics of 38 lakes and ponds on Bathurst Island, Nunavut, Canadian High Arctic. Internat. Rev. Hydrobiol., 86: 1-22.

Mann, M.E., R.S. Bradley, \& M.K. Hughes. 1999. Northern Hemisphere temperatures during the past millennium: inferences, uncertainties, and limitations. Geophys. Res. Letters, 26: 759-762.

McNeely, R.N., V.P. Neimanis \& L. Dwyer. 1979. Water quality sourcebook: A guide to water quality parameters. Water Quality Branch, Inland Waters Directorate, Envi- ronment Canada, Ministry of Supply and Services, Ottawa, Canada: 88 pp.

Meili, M. 1992. Sources, concentrations and characteristics of organic matter in softwater lakes and streams of the Swedish forest region. Hydrobiologia, 229: 23-41.

Moser, K.A., J.P. Smol, D.R.S. Lean \& G.M. MacDonald. 1998. Physical and chemical limnology of northern boreal lakes, Wood Buffalo National Park, northern Alberta and the Northwest Territories, Canada. Hydrobiologia, 377: 25-43.

Overpeck, J., K. Hughen, D. Hardy, R. Bradley, R. Case, M. Douglas, B. Finney, K. Gajewski, G. Jacoby, A. Jennings, S. Lamoureux, A. Lasca, G.M. MacDonald, J. Moore, M. Retelle, S. Smith, A. Wolfe, \& G. Zielinski. 1997. Arctic environmental change of the last four centuries. Science, 278: 1251-1256.

Padgham, W.A. \& W.K. Fyson.1992. The Slave Province: A distinct Archean craton. Can. J. Earth Sci., 29: 2072-2086.

Pienitz, R. 1993. Paleoclimate proxy data inferred from freshwater diatoms from the Yukon and the Northwest Territories. Ph.D. Thesis, Queen's University, Kingston, Ontario: 218pp.

Pienitz, R., J.P. Smol \& D.R.S. Lean. 1997a. Physical and chemical limnology of 24 lakes located between Yellowknife and Contwoyto Lake, Northwest Territories (Canada). Can. J. Fish. Aquat. Sci., 54:347-358.

Pienitz, R., J.P. Smol \& D.R.S. Lean. 1997b. Physical and chemical limnology of 59 lakes located between the southern Yukon and the Tuktoyaktuk Peninsula, Northwest Territories (Canada). Can. J. Fish. Aquat. Sci., 54: 330-346.

Psenner, R. \& R. Schmidt.1992. Climate-driven pH control of remote alpine lakes and effects of acid deposition. Nature, 356: 781-783.

Puznicki, W. 1996. An overview of lake water quality in the Slave structural province area Northwest Territories. Prepared for the Department of Indian and Northern Affairs, Canada: 153 pp.

Ritchie, J.C. 1993. Northern Vegetation. In: H.M. French \& O. Slaymaker (Eds), Canada's Cold Environments. McGillQueen's University Press, Montreal and Kingston: 93-116.

Rouse, W.R., M.S.V. Douglas, R.E. Hecky, A.E. Hershey, G.E. Kling, L. Lesack, P. Marsh, M. McDonald, B.J. Nicholson, N.T. Roulet \& J.P. Smol. 1997. Effects of climate change on the freshwaters of arctic and subarctic North America. Hydrolog. Processes, 11: 873-902.

Rühland, K. \& J.P. Smol. 1998. Limnological characteristics of 70 lakes spanning Arctic treeline from Coronation Gulf to Great Slave Lake in the central Northwest Territories, Canada. Int. Rev. ges. Hydrobiol., 83: 183-203.

Rühland, K. \& J.P. Smol. 2002. Freshwater diatoms from the Canadian Arctic treeline and development of paleolimnological inference models. J. Phycol., 38: 249-264.

Rühland, K., J.P. Smol, \& R. Pienitz. (2003). Ecology and spatial distributions of surface-sediment diatoms from 77 lakes in the subarctic Canadian treeline region. Can. J. Bot.: in press.

Sakomoto, M. 1966. Primary production by phytoplankton community in some Japanese lakes and its dependence on lake depth. Arch. Hydrobiol., 62: 1-28.

Schindler, D.W. 1997. Widespread effects of climatic warming on freshwater ecosystems in North America. In: Cushing, C.E. (Ed.), Freshwater Ecosystems and Climate Change in North America - A Regional Assessment. John Wiley and Sons, Chichester, U.K.: 225-249.

Schindler, D.W., S.E. Bayley, P.J. Curtis, B.R. Parker, M.P. Stainton \& C.A. Kelly. 1992. Natural and man-caused factors affecting the abundance and cycling of dissolved organic substances in precambrian shield lakes. Hydrobiologia, 229: 1-21.

Schindler, D.W., S.E. Bayley, B.R. Parker, K.G. Beaty, D.R. Cruikshank, E.J. Fee, E.U. Schindler \& M.P. Stainton. 
1996. The effects of climatic warming on the properties of boreal lakes and streams at the Experimental Lakes Area, northwestern Ontario. Limnol. Oceanogr., 41: 1004-1017.

Schindler, D.W, P.J. Curtis, S.E. Bayley, B.R. Parker, K.G. Beaty \& M.P. Stainton. 1997. Climate-induced changes in the dissolved organic carbon budgets of boreal lakes. Biogeochemistry, 36: 9-28.

Serreze, M.C., J.E. Walsh, F.S. Chapin III, T. Osterkamp, M. Dyurgerov, V. Romanovsky, W.C. Oechel, J. Morison, T. Zhang \& R.G. Barry. 2000. Observational evidence of recent change in the northern high-latitude environment. Climatic Change, 46: 159-207.

Sirois, L. 1992. The transition between boreal forest and tundra. In: Shugart, H.H., R. Leemans \& G.B. Bonan (Eds), $A$ System Analysis of the Global Boreal Forest. Cambridge University Press, Cambridge: 196-215.

Slaughter, C.W., V.Y.E. Glotov, L.A. Viereck \& V.M. Mikhailov. 1995. Boreal forest catchments: research sites for global change at high latitudes. Wat. Air Soil Poll., 82: 351-361.

Smol, J.P. 1983. Paleophycology of a high arctic lake near Cape Herschel, Ellesmere Island. Can. J. Bot., 61: 21952204.

Smol, J.P. 1988. Paleoclimate proxy data from freshwater arctic diatoms. Verh. int. Ver. Limnol., 23: 837-844. ter Braak, C.J.F. \& P. Šmilauer. 1998. CANOCO for Windows (v. 4.0). Centre for Biometry, Wageningen, The Netherlands.

Timoney, K.P., G.H. La Roi, S.C. Zoltai \& A.L. Robinson. 1993. Vegetation communities and plant distributions and their relationships with parent materials in the forest-tundra of northwestern Canada. Ecography, 16: 174-188.

Webster, K.E., A.D. Newell, L.A. Baker \& P.L. Brezonik. 1990. Climatically induced rapid acidification of a softwater seepage lake. Nature, 347: 374-376.

Weckström, J., A. Korhola \& T. Blom. 1997a. Diatoms as quantitative indicators of $\mathrm{pH}$ and water temperature in subarctic Fennoscandian lakes. Hydrobiologia, 347: 171-184.

Weckström, J., A. Korhola \& T. Blom. 1997b. The relationship between diatoms and water temperature in thirty subarctic Fennoscandian lakes. Arct. Alpine Res., 29: 75-92.

Wedel, J.H., A. Smart \& P. Squires. 1990. An overview study of the Yellowknife River Basin, N.W.T. N.W.T. Programs. Inland Waters Directorate Conservation and Protection, Western and Northern Region, Environment Canada: $82 \mathrm{pp}$.

Welch, H.E. \& J.A. Legault. 1986. Precipitation chemistry and chemical limnology of fertilized and natural lakes at Saqvaqjuac, N.W.T. Can. J. Fish. Aquat. Sci., 43: 1104-1134.

Wetzel, R.G. 1983. Limnology. 2nd edition. Saunders Publishing, Philadelphia, PA: 767 pp. 